Boletín de la Sociedad Geológica Mexicana

VOLUMEN 67, NÚM. 2, 2015, P. 123-143

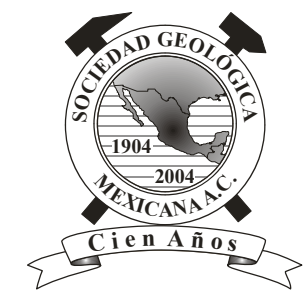

\title{
Geología y estratigrafía del pozo profundo San Lorenzo Tezonco y de sus alrededores, sur de la Cuenca de México
}

\author{
José Luis Arce,,*, Paul Layer², Isela Martínez³, José Iván Salinas4, \\ María del Consuelo Macías-Romoㄹ, Eric Morales-Casique ${ }^{1}$, Jeff Benowitz², Oscar Escolero \\ Nils Lenhardt ${ }^{5}$ \\ ${ }^{1}$ Instituto de Geología, UNAM, Cd. Universitaria Coyoacán, México, D.F., 04510. Tel. 52 + 55-56224288 ext. 108 FAX. 52 + 55- \\ 56224289. \\ ${ }^{2}$ Department of Geology and Geophysics and Geophysical Institute, University of Alaska, Fairbanks, AK, USA. \\ ${ }^{3}$ Escuela Superior de Ingeniería y Arquitectura, IPN, Ticomán, México, D.F. \\ ${ }^{4}$ Facultad de Ingeniería, UNAM, Cd. Universitaria Coyoacán, México, D.F., 04510. \\ ${ }^{5}$ Department of Geology, University of Pretoria, Pretoria 0002, South Africa. \\ *jlarce@geologia.unam.mx
}

\section{Resumen}

La estratigrafía del pozo profundo San Lorenzo Tezonco registra una intensa actividad volcánica en la Cuenca de México y alrededores durante los últimos 20.1 Ma. La columna litológica de 2008 metros de profundidad está dominada por material volcánico, ya sea como flujos de lava o depósitos piroclásticos $(97 \%)$ y solamente los 70 metros más superficiales están constituidos por depósitos lacustres (3\%). Con base en descripciones de campo y análisis de laboratorio (petrografía, geoquímica de roca total y fechamientos radiométricos), fue posible reconocer cuatro paquetes litológicos a lo largo del pozo, los cuales fueron correlacionados con rocas expuestas en superficie. La parte inferior del pozo está representada por rocas pertenecientes a la Formación Tepoztlán (876 - $2008 \mathrm{~m}$ de profundidad) que varían en composición de andesita basáltica a riolita y con edades de 13 a 20.1 Ma. En superficie esta formación aflora alrededor de los poblados de Malinalco y Tepoztlán. Entre las profundidades de 581 y 875 m, se encuentran rocas interpretadas como parte de la Sierra de las Cruces, con una composición de andesita a dacita y edades entre 0.9 y 5 Ma. Hacia la parte superior del pozo $(510-580 \mathrm{~m})$ aparecen rocas volcánicas interpretadas como parte del Cerro de la Estrella, de composición andesítica y fechadas en 0.25 Ma. El último paquete de material volcánico encontrado en el pozo fue interpretado como parte de la Sierra Santa Catarina (70 - $120 \mathrm{~m}$ ) de composición andesítico-basáltica, con edades inferiores a 0.25 Ma, probablemente del Holoceno. Los depósitos lacustres coronan la columna estratigráfica, con edades de hasta 34 mil años.

Adicionalmente, se concluye que las rocas pertenecientes a la Formación Xochitepec, que aflora alrededor de Xochimilco, en la ciudad de México, son de 1.23 a 1.66 Ma de edad, y no del Oligoceno como se había propuesto en trabajos previos. Estas nuevas edades junto con la composición química permiten correlacionar a rocas de Xochitepec con la Sierra de las Cruces.

Palabras clave: Pozo profundo San Lorenzo Tezonco, Cuenca de México, estratigrafía volcánica, geocronología.

\begin{abstract}
The underground stratigraphy of the San Lorenzo Tezonco deep well records intense volcanic activity in the Mexico Basin and surrounding areas during the past $20.1 \mathrm{Ma}$. The lithological column of the $2008 \mathrm{~m}$ deep well is dominated by volcanic material, either as lava flows or pyroclastic deposits (97\%), and only the uppermost $70 \mathrm{~m}$ are composed of lacustrine deposits (3\%). Based on field descriptions and laboratory analyses (petrography, whole-rock chemistry, and radiometric dating), it was possible to recognize four lithological packages throughout the well, which were then correlated with exposed rocks. The lower part of the drill core is represented by rocks belonging to the Tepoztlán Formation (876- $2008 \mathrm{~m}$ depth) that vary in composition from basaltic-andesite to rhyolite, with
\end{abstract}


ages ranging from 13 to $20.1 \mathrm{Ma}$. On the surface this formation outcrops around the towns of Malinalco and Tepoztlán. Between depths of 581 and $875 \mathrm{~m}$, rocks interpreted as being part of the Sierra de las Cruces that vary in composition from andesite to dacite and in age from 0.9 to $5 \mathrm{Ma}$. Upward in the drill core $(510-580 \mathrm{~m})$ are andesitic rocks of Cerro de la Estrella volcano, dated at $0.25 \mathrm{Ma}$. The last volcanic package found in the well is represented by Santa Catarina basaltic-andesitic rocks (70-120 m) that are younger than 0.25 Ma, and probably Holocene. Lacustrine deposits crown the stratigraphic column of the drill core with ages probably younger than 34 ka.

Additionally, we conclude that the rocks belonging to the Xochitepec Formation, outcropping around Xochimilco, in Mexico City, have an age of 1.23 to $1.66 \mathrm{Ma}$, in contrast with the Oligocene age proposed in previous works for these rocks. These new ages plus the chemical composition data allow us to correlate the Xochitepec rocks with those from Sierra de las Cruces

Keywords: San Lorenzo Tezonco deep well, Basin of Mexico, volcanic stratigraphy, geochronology.

\section{Introducción}

El Pozo San Lorenzo Tezonco ( $19^{\circ} 22^{\prime} 44.27^{\prime \prime} \mathrm{N}$ y $99^{\circ}$ $\left.5^{\prime} 5.25^{\prime \prime} \mathrm{O}\right)$ fue perforado en el 2012 por el Sistema de Aguas de la Ciudad de México (SACMEX), en la zona de Iztapalapa, alcanzando una profundidad de 2008 metros. De manera regional, este pozo se ubica dentro de la Cuenca de México, la cual es una depresión de origen volcanotectónico (Enciso-de la Vega, 1992), con una superficie aproximada de $7800 \mathrm{~km}^{2}$, de forma alargada en dirección NE-SO, con un promedio de $100 \mathrm{~km}$ de largo y $30 \mathrm{~km}$ de ancho y una elevación de 2240 metros sobre el nivel del mar (msnm). Esta cuenca abarca parte de los estados de Hidalgo, México, Tlaxcala, Puebla y el Distrito Federal (De Cserna et al., 1988).

A nivel regional la Cuenca de México se encuentra dentro de la provincia geológica del Cinturón Volcánico Trans-Mexicano (Figura 1A), definido como un arco volcánico producido por la subducción de la Placa de Cocos y Rivera por debajo de la placa Norteamericana (Pardo y Suárez, 1995; Ferrari et al., 1999). A nivel local, esta cuenca está limitada por sierras volcánicas conocidas como Sierra de las Cruces al oeste (e.g.: Mora-Alvarez et al., 1991; Osete et al., 2000; Mejia et al., 2005; Arce et al., 2008), la Sierra Chichinautzin al sur (Bloomfield, 1975; Marquez et al., 1999; Siebe et al., 2004; Meriggi et al., 2008), Sierra Nevada al este (Cadoux et al., 2011; Macías et al., 2012) y Sierra de Pachuca y Campo Volcánico Apan-Tezontepec al norte (López-Hernández, 2009; García-Palomo et al., 2002), constituidas por diversas estructuras volcánicas de distintas edades.

El pozo San Lorenzo Tezonco se ubica en la parte sur de la Cuenca de México, a pocos kilómetros al NO de las estructuras volcánicas monogenéticas Sierra de Santa Catarina, Cerro de la Estrella y Peñón del Marqués (Figura 1B). Hacia la parte sur a $15 \mathrm{~km}$ del pozo, inicia el Campo Volcánico Chichinautzin, constituido por una serie de derrames de lava y conos monogenéticos; mientras que hacia la parte oeste, a $25 \mathrm{~km}$ se localiza parte de la Sierra de las Cruces.

A pesar de que solamente se obtuvieron muestras de canal, del pozo profundo, fue posible establecer una columna litológica general (Arce et al., 2013a). Dicha columna litológica está conformada en su parte superior por sedimentos lacustres y fluviales $(0-70 \mathrm{~m}$ de profundidad $)$ y el resto por material volcánico (lavas y depósitos piroclásticos (Arce et al., 2013a), con edades radiométricas reportadas para la parte volcánica entre 0.2 hasta $18 \mathrm{Ma}$ $\left({ }^{40} \mathrm{Ar} /{ }^{39} \mathrm{Ar}\right)(512-1550 \mathrm{~m}$ de profundidad respectivamente), con composiciones de andesita basáltica hasta riolítica (Arce et al., 2013a). Dada la profundidad de este pozo, sin duda representa una oportunidad para establecer la secuencia estratigráfica en el subsuelo de la Cuenca de México y permite hacer correlaciones con unidades volcánicas superficiales.

En este trabajo se complementa la información geocronológica previamente documentada (Arce et al., 2013a) con tres nuevos fechamientos ${ }^{40} \mathrm{Ar} /{ }^{39} \mathrm{Ar}$, de las partes más profundas del pozo ubicadas a 1806, 1920 y $2002 \mathrm{~m}$ de profundidad, se presenta también el análisis químico de 11 muestras de rocas superficiales y su respectiva descripción petrográfica, con el fin de correlacionar las unidades descritas en el pozo con rocas que afloran en los alrededores. También se reportan tres fechamientos radiométricos de muestras de la denominada Formación Xochitepec (Mooser et al., 1956; Colín-Rodríguez, 2006), por el método de U-Pb en circones. Así mismo, se presenta un mapa con la distribución de las rocas volcánicas de los alrededores, construido a partir de descripciones de campo, datos de trabajos previos y modelos digitales de elevación.

\section{Antecedentes geológicos}

Los primeros trabajos sobre la geología y estratigrafía de la Cuenca de México fueron aquellas investigaciones realizadas por Fries $(1956 ; 1960)$, quien llevó a cabo las primeras descripciones de las rocas más antiguas de la región, definiendo nombres formacionales que se siguen utilizando hoy en día. Trabajos posteriores fueron realizados por Mooser $(1963 ; 1975)$ enfocados al estudio de las rocas volcánicas más recientes, sin embargo, también de carácter general.

Después del terremoto de 1985 que destruyó una parte 


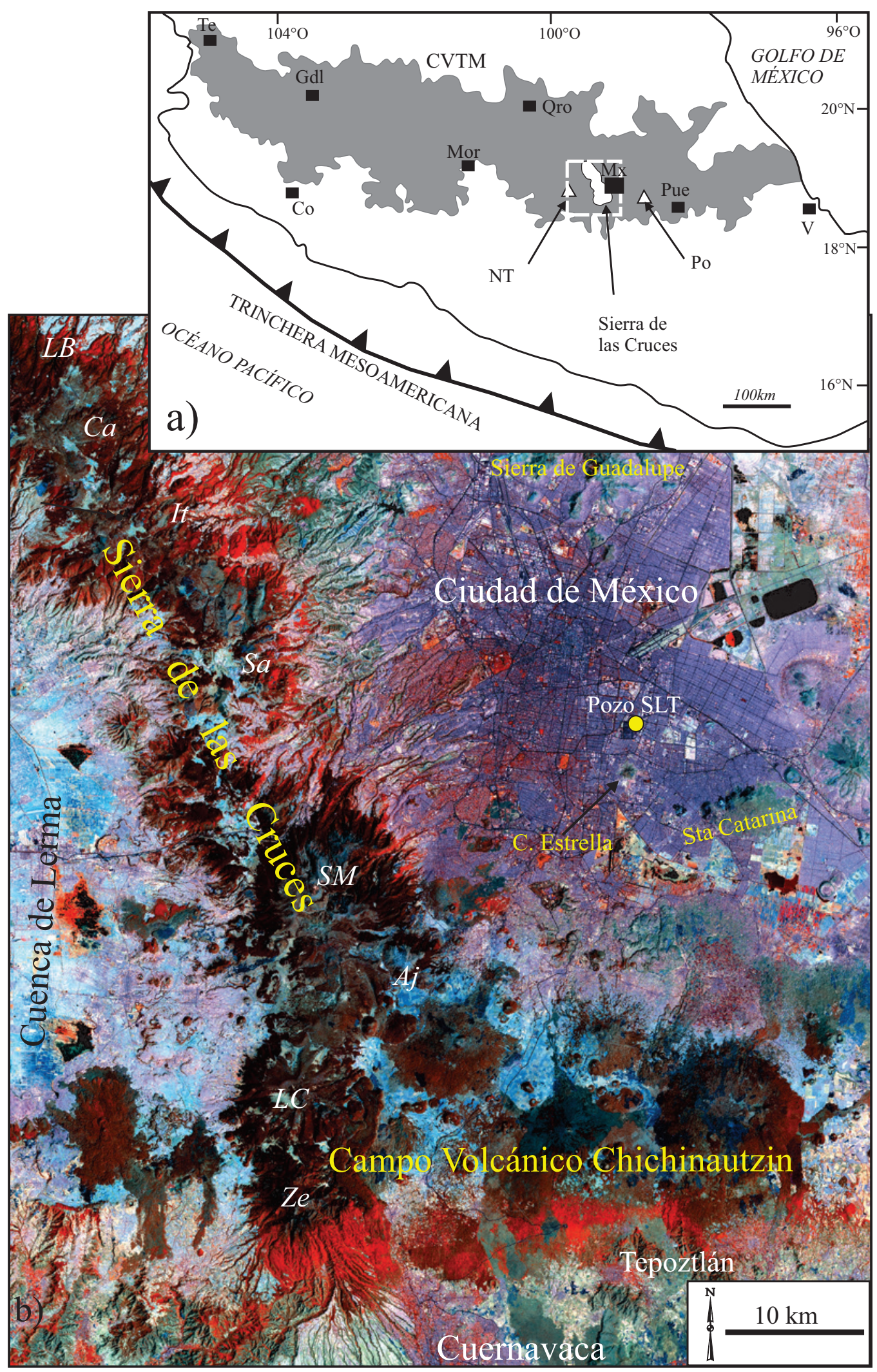

Figura 1. a) Mapa del Cinturón Volcánico Trans-Mexicano, mostrando la ubicación de la Cuenca de México. El recuadro en el CVTM representa el área de la imagen de satélite en el inciso b). Las estructuras volcánicas en a) son: Po, Popocatépetl y NT, Nevado de Toluca; las ciudades de V, Veracruz; Pue, Puebla; Mx, Ciudad de México; Qro, Querétaro; Mor, Morelia; Co, Colima; Gdl, Guadalajara y Te, Tepic. b) Imagen de satélite mostrando la parte sur de la Cuenca de México y los distintos elementos que se discuten en este artículo. Se abrevian las siguientes estructuras volcánicas: LB, La Bufa; Ca, Catedral; It, Iturbide; Sa, Salazar; SM, San Miguel; Aj, Ajusco; LC, La Corona; Ze, Zempoala, así como la ubicación del pozo profundo San Lorenzo Tezonco, SLT. Modificado de Arce et al. (2008). 
importante de la ciudad de México, se realizó un proyecto para entender la estructura geológica, gravimétrica y neotectónica de la Cuenca de México (De Cserna et al., 1988) misma que hasta la fecha es un material de referencia obligado, debido en parte a que no se han llevado a cabo trabajos integrales recientes. Posteriormente VázquezSánchez y Jaimes-Palomera (1989) propusieron una nomenclatura estratigráfica para las rocas del subsuelo de la Cuenca de México, así como para rocas que afloran en los alrededores, sin duda también representa un trabajo importante desde el punto de vista geológico.

Adicionalmente, en la Cuenca de México se perforaron pozos profundos en los años 80's cuyos cortes litológicos han sido utilizados para interpretar la geología del subsuelo (Pérez-Cruz, 1988), aunque con mucha incertidumbre debido a que en su mayoría se trata de muestras de canal, cuyas descripciones fueron hechas por diversas personas y en distintos tiempos, siguiendo criterios diversos. Además, los fechamientos radiométricos que se llevaron a cabo con algunas muestras de los pozos profundos fueron mediante el método de $\mathrm{K}-\mathrm{Ar}$, que resultan comúnmente sobreestimados o con gran incertidumbre, debido a la alteración de las rocas.

Más recientemente el Sistema de Aguas de la Ciudad de México (SACMEX) dependiente del Gobierno del D.F., ordenó perforar un pozo profundo en la zona de Iztapalapa, el cual alcanzó una profundidad de 2008 metros, con muestreo a cada 2 metros, pero sin recuperación de núcleos, por lo que la estratigrafía que se ha obtenido de éste es general, ya que sólo fue posible determinar cambios litológicos a escala métrica (Arce et al., 2013a). En general, la edad más antigua en este pozo fue de 18 Ma y corresponde a una profundidad de $1550 \mathrm{~m}$. Además, estos mismos autores propusieron que el Pozo San Lorenzo Tezonco se ubica en una estructura de graben orientado en dirección $\mathrm{E}-\mathrm{O}$ y coincide con la orientación de la Sierra de Santa Catarina, conformada por seis volcanes monogenéticos.

Otro tipo de estudios que se han llevado a cabo sobre la Cuenca de México han involucrado núcleos de pozos, pero con profundidades de sólo $30 \mathrm{~m}$, considerando solamente la parte lacustre. Estos trabajos han aportado datos sobre la estratigrafía del Holoceno y Pleistoceno tardío (LozanoGarcía y Ortega-Guerrero, 1998; Caballero y OrtegaGuerrero, 1998), así como información paleoambiental, incluyendo fechamientos por el método de radiocarbono. Las edades más viejas que se han publicado de estos pozos someros oscilan entre 10 y 220 mil años antes del presente (A.P.) (Lozano-García et al., 1993; Lozano-García y OrtegaGuerrero, 1998; Brown et al., 2012), lo cual representa una mínima parte para todo el paquete volcánico que se ha reportado en los pozos profundos.

También se cuenta con una serie de estudios aislados de las distintas sierras volcánicas que bordean a la Cuenca de México, los cuales han sido publicados en diferentes tiempos, incluyendo mapas locales de estructuras volcánicas (Osete et al., 2000; Siebe et al., 2004; Arce et al., 2008; García-Palomo et al., 2008; Cadoux et al., 2011; Macías et al., 2012) o estudios de eventos volcánicos particulares (Siebe, 2000; Agustín-Flores et al., 2011; Rueda et al., 2013).

\section{Metodología}

La metodología que se empleó para el presente trabajo consistió de dos partes fundamentales, trabajo de campo y análisis de laboratorio. Para el trabajo de campo en primer lugar se realizó un modelo digital de elevación de la parte sur de la Cuenca de México, con el fin de determinar características morfológicas de las distintas sierras, al sur y suroeste del Pozo San Lorenzo Tezonco, posteriormente se elaboró un mapa fotogeológico con ayuda de modelos digitales de elevación y fotografías aéreas. Esto fue complementado con los trabajos previos (Martin del Pozzo, 1982; Osete et al., 2000; Siebe, 2000; Siebe et al., 2004; García-Palomo et al., 2008) y una vez que el mapa geológico preliminar estuvo listo, se procedió a realizar el trabajo de campo. En el trabajo de campo se realizaron descripciones de distintos afloramientos, en particular del tipo de roca y su composición mineralógica. Al mismo tiempo se llevó a cabo un muestreo de rocas para distintos análisis de laboratorio. En campo también se modificó el mapa fotogeológico de acuerdo a las descripciones de los afloramientos.

Los análisis de laboratorio consistieron en diversas técnicas, para determinar la composición química de elementos mayores y trazas por los métodos de FRX e ICPMS; fechamientos radiométricos por los métodos ${ }^{40} \mathrm{Ar} /{ }^{39} \mathrm{Ar} y$ $\mathrm{U}-\mathrm{Pb}$ en circones, mismos que se describen a continuación.

\subsection{FRX e ICP-MS}

En total se colectaron en el campo 11 muestras, de las cuales se elaboraron láminas delgadas. Estas láminas fueron descritas con ayuda de un microscopio petrográfico, poniendo énfasis en la textura, asociación mineralógica y características de cada fase mineral. Las mismas 11 muestras de roca fueron pulverizadas, con el fin de llevar a cabo análisis químicos de roca total, por el método de Fluorescencia de Rayos X (FRX), en el Laboratorio Universitario de Geoquímica Isotópica (LUGIS, UNAM) para determinar elementos mayores y pérdida por calcinación (Tabla 1) y por el método de Inductively Coupled Plasma Mass Spectrometry (ICP-MS) para determinar elementos traza (Tabla 1), el cual se llevó a cabo en el Centro de Geociencias, UNAM, en Juriquilla, Querétaro. Las metodologías detalladas están descritas en Lozano-Santacruz y Bernal (2005) para FRX y Mori et al. (2007) para ICP-MS.

\subsection{Geocronología por ${ }^{40} \mathrm{Ar} /{ }^{39} \mathrm{Ar}$}

Con el objetivo de extender el registro geocronológico existente (Arce et al., 2013a), se enviaron tres muestras 
Tabla 1. Análisis químicos de roca total de muestras del sur de la Cuenca de México. Mapas de las Figs. 6 y 10 muestran la localización de las muestras analizadas.

\begin{tabular}{|c|c|c|c|c|c|c|c|c|c|c|c|}
\hline $\begin{array}{l}\text { Localidad } \\
\text { Muestra }\end{array}$ & $\begin{array}{c}\text { C Estrella } \\
\text { CE-1302 } \\
\end{array}$ & $\begin{array}{c}\text { C Estrella } \\
\text { CE-1303 } \\
\end{array}$ & $\begin{array}{l}\text { S. Catarina } \\
\text { SC-1304 }\end{array}$ & $\begin{array}{c}\text { Sn Miguel } \\
\text { SM-1302 }\end{array}$ & $\begin{array}{c}\text { Sn Miguel } \\
\text { SM-1332 } \\
\end{array}$ & $\begin{array}{c}\text { V Tezontle } \\
\text { SM-1310 }\end{array}$ & $\begin{array}{c}\text { Xochitepec } \\
\text { X01204 } \\
\end{array}$ & $\begin{array}{r}\text { Xochitepe } \\
\text { X01205 } \\
\end{array}$ & $\begin{array}{c}\text { Xochitepec } \\
\text { X01206 } \\
\end{array}$ & $\begin{array}{c}\text { dique } \\
\text { X01207 } \\
\end{array}$ & $\begin{array}{c}\text { Xochitepec } \\
\text { X01210 } \\
\end{array}$ \\
\hline$\overline{\mathrm{SiO}_{2}}$ & 58.15 & 58.38 & 57.20 & 63.20 & 61.65 & 56.42 & 65.27 & 64.06 & 66.73 & 54.79 & 66.71 \\
\hline $\mathrm{TiO}_{2}$ & 1.26 & 1.24 & 1.35 & 0.81 & 0.75 & 1.21 & 0.62 & 0.68 & 0.55 & 1.23 & 0.63 \\
\hline $\mathrm{Al}_{2} \mathrm{O}_{3}$ & 16.47 & 16.16 & 15.90 & 16.62 & 15.80 & 15.87 & 16.11 & 16.79 & 16.35 & 15.76 & 16.02 \\
\hline $\mathrm{Fe}_{2} \mathrm{O}_{3 \mathrm{t}}$ & 7.34 & 7.27 & 8.09 & 5.42 & 5.16 & 7.39 & 4.40 & 4.69 & 3.85 & 7.58 & 4.32 \\
\hline $\mathrm{MnO}$ & 0.12 & 0.12 & 0.14 & 0.09 & 0.07 & 0.12 & 0.07 & 0.08 & 0.05 & 0.11 & 0.07 \\
\hline $\mathrm{MgO}$ & 4.45 & 4.32 & 4.37 & 2.63 & 3.22 & 6.18 & 1.71 & 2.10 & 0.84 & 6.03 & 0.72 \\
\hline $\mathrm{CaO}$ & 6.03 & 6.11 & 6.84 & 4.84 & 5.33 & 6.82 & 4.34 & 4.40 & 3.80 & 7.19 & 3.89 \\
\hline $\mathrm{Na}_{2} \mathrm{O}$ & 3.37 & 3.53 & 4.05 & 4.64 & 4.31 & 4.19 & 4.41 & 4.54 & 4.62 & 3.64 & 4.71 \\
\hline $\mathrm{K} 2 \mathrm{O}$ & 2.10 & 2.11 & 1.74 & 1.61 & 1.66 & 1.62 & 1.85 & 1.77 & 1.87 & 1.81 & 1.94 \\
\hline $\mathrm{P}_{2} \mathrm{O}_{5}$ & 0.35 & 0.36 & 0.58 & 0.15 & 0.20 & 0.35 & 0.19 & 0.20 & 0.14 & 0.46 & 0.15 \\
\hline $\mathrm{P} \times \mathrm{C}$ & 0.00 & 0.25 & -0.34 & -0.03 & 1.85 & -0.07 & 1.14 & 0.21 & 1.00 & 1.30 & 0.91 \\
\hline Total & 99.64 & 99.85 & 99.91 & 99.98 & 100.00 & 100.09 & 100.11 & 99.53 & 99.80 & 99.89 & 100.09 \\
\hline $\mathrm{Li}$ & 13.80 & 14.26 & 17.24 & 15.06 & 11.85 & 16.26 & 32.06 & 32.06 & 22.53 & 14.77 & 20.54 \\
\hline $\mathrm{Be}$ & 2.13 & 2.08 & 2.18 & 1.45 & 1.52 & 1.80 & 1.67 & 1.63 & 1.74 & 1.51 & 1.46 \\
\hline $\mathrm{Sc}$ & 16.43 & 17.11 & 15.77 & 11.64 & 12.78 & 16.05 & 9.72 & 10.45 & 7.54 & 17.66 & 8.50 \\
\hline V & 114.94 & 120.07 & 116.95 & 84.77 & 100.04 & 119.06 & 71.52 & 72.84 & 59.30 & 138.43 & 68.92 \\
\hline $\mathrm{Cr}$ & 138.50 & 146.92 & 147.10 & 70.50 & 79.02 & 275.52 & 59.50 & 77.85 & 102.22 & 236.92 & 62.12 \\
\hline Co & 20.03 & 21.00 & 20.36 & 12.98 & 14.19 & 28.06 & 11.07 & 12.10 & 9.00 & 27.26 & 10.07 \\
\hline $\mathrm{Ni}$ & 37.95 & 42.15 & 45.82 & 28.90 & 18.00 & 140.40 & 21.10 & 29.35 & 18.64 & 128.72 & 16.84 \\
\hline $\mathrm{Cu}$ & 15.15 & 20.15 & 22.76 & 15.39 & 17.20 & 32.96 & 11.95 & 9.84 & 12.37 & 40.98 & 15.73 \\
\hline $\mathrm{Zn}$ & 84.16 & 89.93 & 94.04 & 69.59 & 64.34 & 73.80 & 62.88 & 63.65 & 59.02 & 83.44 & 64.28 \\
\hline $\mathrm{Ga}$ & 19.81 & 20.81 & 19.98 & 19.78 & 19.13 & 18.39 & 19.12 & 19.81 & 20.12 & 18.39 & 20.37 \\
\hline $\mathrm{Rb}$ & 45.38 & 47.92 & 36.68 & 29.31 & 29.72 & 27.93 & 47.12 & 38.58 & 46.89 & 32.39 & 46.17 \\
\hline $\mathrm{Sr}$ & 395.42 & 415.53 & 512.54 & 469.65 & 630.89 & 676.87 & 519.22 & 550.18 & 451.55 & 1083 & 461.99 \\
\hline Y & 31.25 & 32.78 & 33.26 & 18.28 & 18.45 & 23.47 & 16.93 & 17.84 & 15.02 & 24.74 & 14.36 \\
\hline $\mathrm{Zr}$ & 349.41 & 359.64 & 387.25 & 138.11 & 107.25 & 188.71 & 88.85 & 112.79 & 24.49 & 214.85 & 32.71 \\
\hline $\mathrm{Nb}$ & 17.86 & 18.56 & 23.27 & 5.17 & 3.94 & 13.26 & 5.36 & 5.28 & 4.67 & 7.94 & 5.38 \\
\hline Mo & 2.20 & 2.47 & 3.04 & 1.11 & 0.66 & 2.53 & 1.08 & 1.59 & 0.60 & 0.52 & 0.27 \\
\hline Sn & 1.63 & 1.70 & 1.43 & 0.85 & 0.88 & 1.20 & 1.04 & 1.11 & 0.94 & 1.19 & 1.09 \\
\hline $\mathrm{Sb}$ & 0.14 & 0.15 & 0.23 & 0.08 & 0.11 & 0.10 & 0.30 & 0.21 & 0.14 & 0.14 & 0.10 \\
\hline Cs & 1.27 & 1.34 & 0.98 & 0.71 & 0.77 & 1.06 & 1.69 & 1.48 & 1.00 & 1.17 & 1.42 \\
\hline $\mathrm{Ba}$ & 592.63 & 628.90 & 537.98 & 368.44 & 386.55 & 559.38 & 560.83 & 571.22 & 473.07 & 787.49 & 481.92 \\
\hline $\mathrm{La}$ & 34.77 & 35.33 & 39.55 & 12.64 & 19.00 & 27.18 & 18.70 & 20.92 & 17.30 & 39.28 & 15.59 \\
\hline $\mathrm{Ce}$ & 71.54 & 74.53 & 82.93 & 26.52 & 38.75 & 59.69 & 40.92 & 45.99 & 30.00 & 85.88 & 34.66 \\
\hline $\operatorname{Pr}$ & 9.24 & 9.48 & 10.45 & 3.79 & 5.75 & 8.14 & 5.28 & 6.23 & 4.91 & 11.69 & 4.13 \\
\hline $\mathrm{Nd}$ & 35.76 & 36.79 & 40.36 & 16.18 & 23.82 & 33.47 & 21.60 & 25.20 & 19.85 & 47.35 & 16.54 \\
\hline Sm & 7.31 & 7.57 & 8.03 & 3.82 & 5.06 & 7.02 & 4.53 & 5.30 & 4.57 & 9.07 & 3.76 \\
\hline $\mathrm{Eu}$ & 1.76 & 1.82 & 2.04 & 1.12 & 1.36 & 1.92 & 1.23 & 1.40 & 1.11 & 2.26 & 1.04 \\
\hline $\mathrm{Tb}$ & 0.98 & 1.02 & 1.06 & 0.57 & 0.61 & 0.82 & 0.56 & 0.63 & 0.55 & 0.91 & 0.48 \\
\hline $\mathrm{Gd}$ & 6.59 & 6.83 & 7.14 & 3.73 & 4.32 & 5.87 & 3.90 & 4.40 & 3.83 & 6.78 & 3.31 \\
\hline Dy & 5.72 & 5.95 & 6.03 & 3.31 & 3.42 & 4.43 & 3.04 & 3.35 & 2.98 & 4.73 & 2.59 \\
\hline Но & 1.12 & 1.16 & 1.18 & 0.65 & 0.66 & 0.84 & 0.59 & 0.64 & 0.56 & 0.87 & 0.50 \\
\hline $\mathrm{Er}$ & 3.09 & 3.23 & 3.24 & 1.78 & 1.75 & 2.27 & 1.54 & 1.65 & 1.45 & 2.29 & 1.30 \\
\hline $\mathrm{Yb}$ & 2.89 & 3.02 & 3.02 & 1.66 & 1.62 & 2.05 & 1.40 & 1.50 & 1.30 & 2.07 & 1.16 \\
\hline $\mathrm{Lu}$ & 0.43 & 0.44 & 0.45 & 0.25 & 0.24 & 0.30 & 0.21 & 0.22 & 0.19 & 0.30 & 0.18 \\
\hline $\mathrm{Hf}$ & 6.93 & 7.14 & 7.06 & 3.52 & 2.93 & 4.21 & 2.12 & 2.54 & 0.71 & 4.52 & 0.97 \\
\hline $\mathrm{Ta}$ & 1.02 & 1.05 & 1.23 & 0.35 & 0.29 & 0.81 & 0.38 & 0.38 & 0.35 & 0.49 & 0.40 \\
\hline W & 2.26 & 2.47 & 3.09 & 1.62 & 0.59 & 3.93 & 1.46 & 1.49 & 3.87 & 1.54 & 1.67 \\
\hline $\mathrm{Tl}$ & 0.28 & 0.29 & 0.16 & 0.16 & 0.05 & 0.14 & 0.09 & 0.17 & 0.22 & 2.40 & 0.10 \\
\hline $\mathrm{Pb}$ & 8.89 & 10.17 & 8.09 & 5.91 & 6.85 & 7.14 & 8.36 & 8.62 & 7.51 & 9.20 & 7.65 \\
\hline Th & 5.36 & 5.58 & 4.32 & 2.46 & 3.22 & 3.70 & 3.86 & 3.85 & 2.59 & 3.49 & 4.03 \\
\hline $\mathrm{U}$ & 1.26 & 1.31 & 1.33 & 0.90 & 1.08 & 1.30 & 1.60 & 1.40 & 0.72 & 1.30 & 0.77 \\
\hline
\end{tabular}

Los valores están reportados en \% en peso (elementos mayores) y ppm (elementos traza). PxC, pérdida por calcinación; * $\mathrm{Fe}_{\text {total reportado como } \mathrm{Fe}} \mathrm{O}_{3}$. 
del pozo profundo San Lorenzo Tezonco, al Laboratorio de Geocronología de la Universidad de Alaska, Fairbanks, E.U.A., para llevar a cabo fechamientos radiométricos con el método de ${ }^{40} \mathrm{Ar} /{ }^{39} \mathrm{Ar}$ (Figura 4 y Tabla 2) y de esta manera complementar el registro geocronológico del pozo profundo. Estos tres nuevos fechamientos corresponden con las muestras más profundas del pozo San Lorenzo Tezonco, ubicadas a 1806, 1920 y 2002 metros de profundidad.

Los fechamientos se llevaron a cabo por calentamiento a pasos por láser, de la roca total (matriz de la muestra) siguiendo los procedimientos descritos en Layer (2000) y Layer et al. (2009a). Para monitorear el flujo de neutrón y calcular el parámetro de irradiación "J" para todas las muestras se utilizó el mineral MMhb-1T, con una edad de 523.5 Ma (Renne et al., 2010). Los isótopos de argón medidos fueron corregidos por un sistema de blancos y discriminación de masas, mientras que para las muestras irradiadas, las reacciones de interferencia de calcio, potasio y cloro se determinaron siguiendo los procedimientos descritos en McDougall y Harrison (1999) y las edades fueron calculadas usando las constantes de Renne et al. (2010) y están reportadas con valor de 1-sigma.

\subsection{Geocronología U-Pb en circones}

También se realizaron fechamientos por el método $\mathrm{U}-\mathrm{Pb}$ en circones, de tres muestras de la denominada Formación Xochitepec (Tabla 3). De la muestra XO-1204 se analizaron en total 20 cristales de circón; mientras que de la muestra XO-1205 se analizaron en total 18 cristales y por último, de la muestra XO-1210 se analizaron 26 cristales (Tabla 3). Los cristales de circón se montaron en portaobjetos de vidrio y se pulieron para ser analizados por espectrómetro de masas con acoplamiento inductivo con ablación láser (LA-ICP-MS, por sus siglas en inglés) siguiendo la metodología descrita en Solari et al. (2010) para geocronología por U-Pb. Estos análisis se llevaron a cabo en el Laboratorio de Estudios Isotópicos del Centro de Geociencias, UNAM, Juriquilla,

Tabla 2. Resumen de análisis ${ }^{40} \mathrm{Ar} /{ }^{39} \mathrm{Ar}$ de muestras del pozo profundo San Lorenzo Tezonco.

\begin{tabular}{|c|c|c|c|}
\hline $\begin{array}{c}\text { Muestra } \\
\text { (profundidad) }\end{array}$ & $\begin{array}{c}\text { Edad de la } \\
\text { isócrona (Ma) }\end{array}$ & $\begin{array}{l}\text { Información de la } \\
\text { isócrona }\end{array}$ & $\begin{array}{c}\text { Edad de la meseta } \\
\text { (Ma) }\end{array}$ \\
\hline 1806 & $17.5 \pm 0.6$ & $\begin{array}{c}7 \text { fracciones de } 7 \\
{ }^{40} \mathrm{Ar}^{36} \mathrm{Ar}_{\mathrm{i}}=298.7 \pm 2.1 \\
\mathrm{MSWD}=3.71\end{array}$ & $17.0 \pm 0.8$ \\
\hline 1920 & $18.3 \pm 0.4$ & $\begin{array}{c}7 \text { fracciones de } 7 \\
{ }^{40} \mathrm{Ar}^{36}{ }^{\mathrm{Ar}_{\mathrm{i}}}=304.2 \pm 1.5 \\
\mathrm{MSWD}=1.66\end{array}$ & $18.9 \pm 0.4$ \\
\hline 2002 & $20.1 \pm 0.3$ & $\begin{array}{c}7 \text { fracciones de } 7 \\
{ }^{40} \mathrm{Ar}^{36}{ }^{\mathrm{Ar}_{\mathrm{i}}}=304.8 \pm 2.1 \\
\mathrm{MSWD}=0.49\end{array}$ & $20.5 \pm 0.3$ \\
\hline
\end{tabular}

Para cada muestra se analizaron de $2-4$ alícuotas, por calentamiento a pasos por láser (corridas). Las alícuotas consistieron de 1 a 5 fragmentos pequeños de las muestras de canal. Todas las edades están reportadas con incertidumbre de \pm 1 -sigma, calculada usando las constantes de Renne et al. (2010). MSWD: Desviación ponderada media cuadrática.
Querétaro, México.

De acuerdo con los patrones de tierras raras de cada circón analizado, todos ellos corresponden con patrones de rocas magmáticas, de modo que las edades correspondientes a la denominada Formación Xochitepec, corresponderían a edades máximas.

\section{Resultados}

En esta sección se describen, de manera resumida, las características del pozo profundo San Lorenzo Tezonco, el cual ha sido analizado de manera detallada en otros trabajos (Arce et al., 2013a y Morales-Casique et al., 2014), así como la descripción de las distintas estructuras y/o sierras volcánicas aledañas al pozo, enfatizando sus características petrográficas, químicas y distribución de sus productos.

\subsection{Pozo San Lorenzo Tezonco}

Con base en la información disponible de muestras del pozo, datos presentados en este trabajo, en particular los fechamientos ${ }^{40} \mathrm{Ar} /{ }^{39} \mathrm{Ar}$ y la composición química de muestras superficiales, en el presente trabajo se dividió a la estratigrafía del pozo en los siguientes paquetes: 1) Lacustre (0 - 70 metros); 2) Santa Catarina y Cerro de la Estrella (71 - 580 metros); 3) Sierra de las Cruces (581 - 875 metros); y 4) Formación Tepoztlán (876 - 2008 metros) (Figura 2). El paquete Lacustre consta de depósitos epiclásticos (arenas, limos y arcillas), en algunos niveles se observaron conchas de ostrácodos. El espesor de los depósitos lacustres es variable, en algunos sitios se han perforado 11 metros, con edad máxima de > 34 mil años (Lozano-García et al., 1993; Lozano-García y Ortega-Guerrero, 1998), en el área de Texcoco se han reportado espesores de $80 \mathrm{~m}$, con una edad máxima probable de 220 mil años (Lozano-García, 1989) y recientemente se han reportado espesores de hasta 122 metros con edades preliminares de 220 mil años, en la zona de Chalco (Brown et al., 2012).

El paquete Santa Catarina y Cerro de la Estrella, consiste de dos tipos de lava, ambos de color gris oscuro, de composición andesítico-basáltica y andesítica (Figura 3), con pocos fenocristales de plagioclasa, piroxeno y en menor proporción, olivino, cuya edad no muy precisa, es de $\leq 0.25$ Ma (Figura 2), correlacionado con productos del Campo Volcánico Chichinautzin (Arce et al., 2013a). Sin embargo en este trabajo se plantea que estas lavas podrían pertenecer a productos de la Sierra de Santa Catarina y Cerro de la Estrella, con base en: 1) la cercanía de entre 4 y $7 \mathrm{~km}$ con respecto al Pozo San Lorenzo Tezonco; 2) La composición química (ver secciones posteriores); y 3) la edad ${ }^{40} \mathrm{Ar} /{ }^{39} \mathrm{Ar}$ muy joven sugerida para Santa Catarina (probablemente del Holoceno) (Layer et al., 2009b).

A continuación se tiene al paquete Sierra de las Cruces (Figura 2), que consiste de lavas de color gris claro, de textura porfídica con fenocristales de plagioclasa, anfíbol 
y piroxeno (lava dacítica), con edades entre 0.9 y $1.8 \mathrm{Ma}$ (Arce et al., 2013a) correlacionada con productos de la Sierra de las Cruces, ubicada al occidente de la Cuenca de México. Para este paquete también se tiene una secuencia de depósitos de ignimbrita, parcialmente alterada a arcilla, con abundantes cristales de plagioclasa, anfíbol, cuarzo y en menor proporción piroxeno, que corresponde a una composición riolítica y fechada en $5 \mathrm{Ma}$ (Arce et al., 2013a) y abarca las profundidades de 760 a 875 metros.

Con base en los nuevos fechamientos ${ }^{40} \mathrm{Ar} /{ }^{39} \mathrm{Ar}$

Tabla 3. Resumen de los análisis de U-Pb en circones de rocas de la Formación Xochitepec, Xochimilco, D.F. Mapas de las Figs. 6 y 10 muestran la localización de las muestras analizadas.

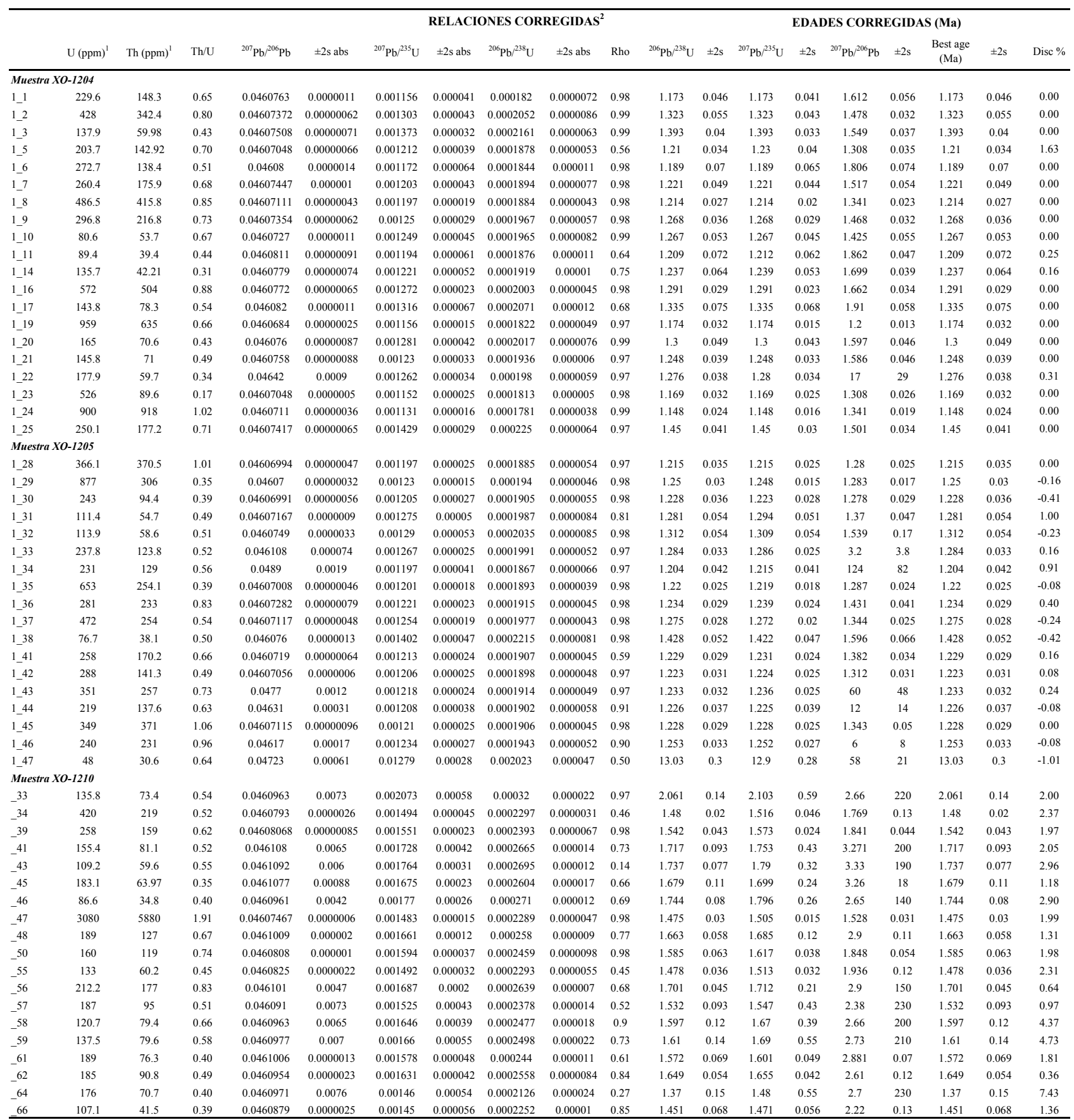

1: Concentraciones de U y Th fueron calculadas usando un circón estándar externo de acuerdo a Paton et al. (2010), Geochemistry, Geophysics, Geosystems. 2: Incertidumbre de 2 sigma propagado de acuerdo a Paton et al. (2010), Geochemistry, Geophysics, Geosystems.

Relaciones de ${ }^{207} \mathrm{~Pb} / 206 \mathrm{~Pb}$, edades y errores fueron calculadas de acuerdo a Petrus y Kamber (2012), Geostandards Geoanalytical Research.

El área analizada fue de 23 micras, usando un protocolo analítico modificado de Solari et al. (2010), Geostandards Geoanalytical Research.

Los datos fueron medidos empleando un MC-ICPMS-Neptune Plus, acoplado a un sistema de ablación láser Resolution M050 Excimer. 


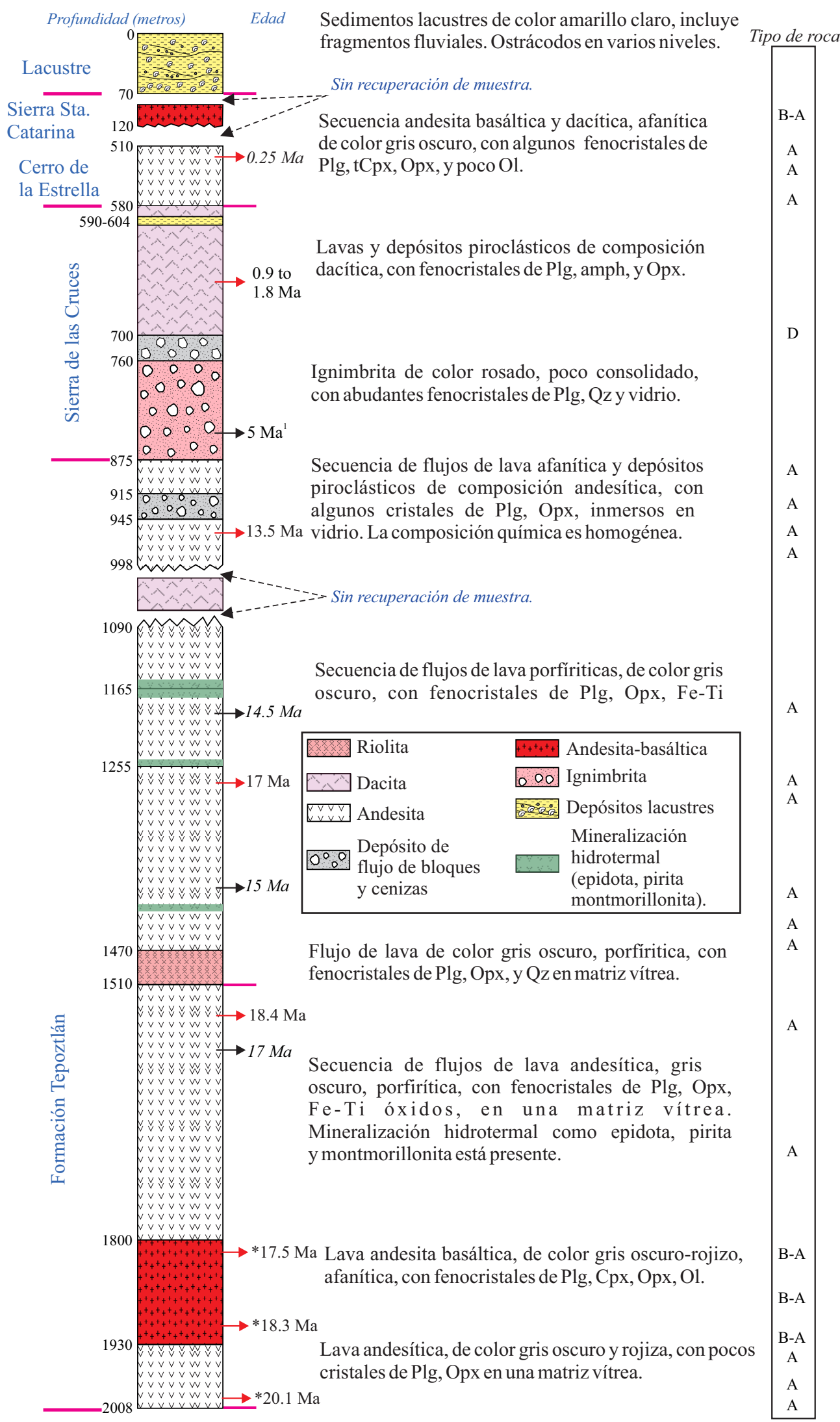

Figura 2. Columna estratigráfica del pozo profundo San Lorenzo Tezonco. El tipo de roca está basado en análisis químico de roca total (A, andesita; D, dacita; B-A, andesita-basáltica). Las edades con asterisco son de este trabajo (Tabla 2). Las edades retomadas de Arce et al. (2013a) son de U-Pb en circones y las demás son ${ }^{40} \mathrm{Ar}{ }^{/ 39} \mathrm{Ar}$. La separación en paquetes se llevó a cabo con base en la edad, composición química y relaciones de campo (ver la discusión en el texto). Modificado de Arce et al. (2013a). 

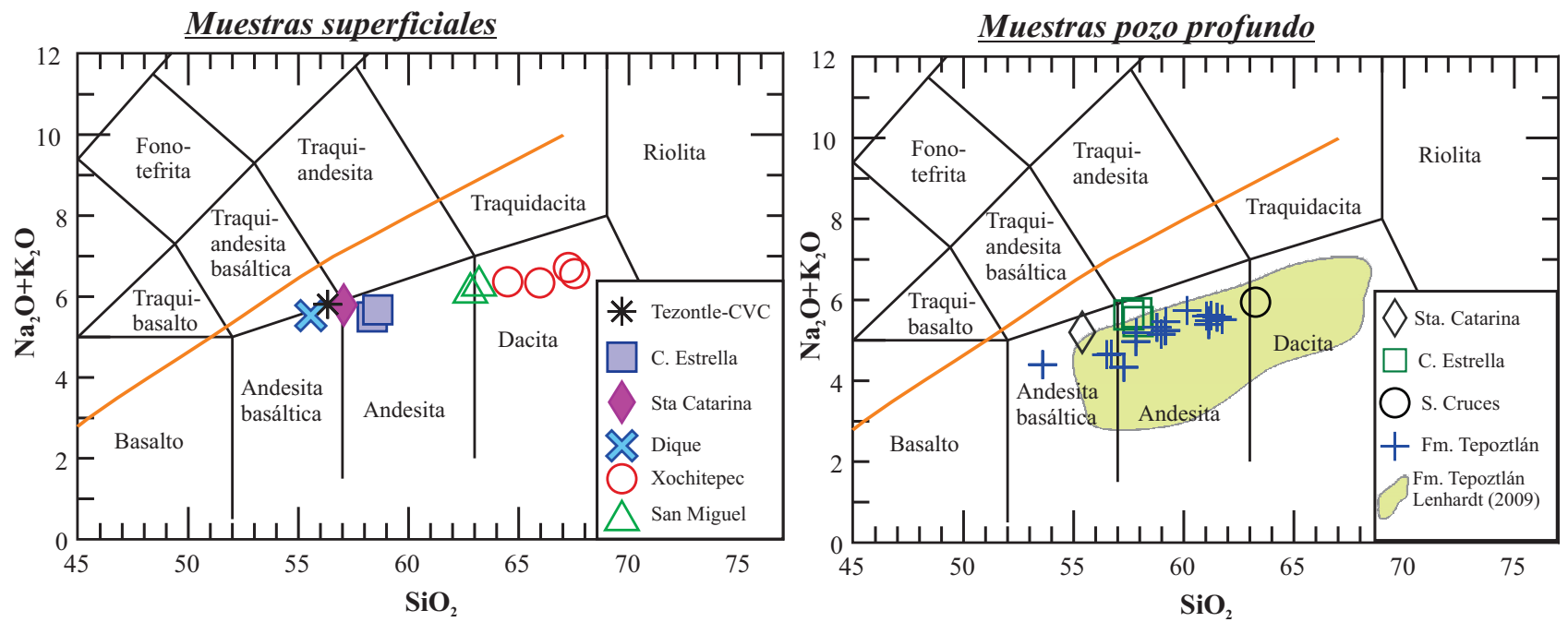

Figura 3. Diagrama álcalis total vs. sílice (Le Bas et al., 1986) para la clasificación química de las rocas del pozo profundo y de muestras superficiales reportadas en este trabajo. El volcán Tezontle es parte del Campo Volcánico Chichinautzin (CVC). Se muestra también la línea que divide a los campos alcalinos y calci-alcalinos de acuerdo con Macdonald y Katsura (1964). Los datos graficados están normalizados en base anhidra.

reportados en el presente trabajo (Tabla 2), se propone un último paquete de rocas correlacionado con la Formación Tepoztlán, que abarca desde los 875 metros hasta el final del pozo (2008 metros de profundidad). Este paquete consiste de una secuencia de lavas y en menor cantidad depósitos piroclásticos, de composición diversa, de andesita basáltica, andesita, dacita y riolita (Figura 3) similar a rocas descritas para la Formación Tepoztlán, la cual aflora aproximadamente a $40 \mathrm{~km}$ al sur del pozo San Lorenzo Tezonco, en la localidad de Tepoztlán, Morelos, con edades entre 11 y $22.8 \mathrm{Ma}$ (Lenhardt et al., 2010).

Los nuevos fechamientos de ${ }^{40} \mathrm{Ar} /{ }^{39} \mathrm{Ar}$ reportados en este trabajo, complementan la información geocronológica previamente documentada (Arce et al., 2013a) del pozo San Lorenzo Tezonco, en particular de las rocas más profundas. La muestra ubicada a $1806 \mathrm{~m}$, arrojó una edad de $17.5 \mathrm{Ma}$; mientras que la muestra de $1920 \mathrm{~m}$ registró una edad de 18.3 Ma y la más profunda ubicada a $2002 \mathrm{~m}$, arrojó una edad de 20.1 Ma (Figura 4; Tabla 2).

\subsection{Sierra de las Cruces y Fm Xochitepec}

La Sierra de las Cruces constituye el límite occidental de la cuenca de México (Figura 1B), constituida por ocho estructuras volcánicas traslapadas (García-Palomo et al., 2008). De sur a norte, se han reconocido los siguientes estratovolcanes: Zempoala (3690 m.s.n.m.), La Corona (3770 m.s.n.m.), San Miguel (3870 m.s.n.m.), Salazar (3660 m.s.n.m.), Chimalpa (3420 m.s.n.m.), Iturbide (3620 m.s.n.m.), La Bufa (3460 m.s.n.m.) y La Catedral (3780 m.s.n.m.), así como otras estructuras de menor volumen como el volcán Ajusco. La actividad volcánica en esta sierra registró una migración de norte a sur, ya que las edades más antiguas se han reportado en el volcán La Bufa, de 3.7 Ma (Aguirre-Díaz et al., 2006) y las edades más recientes corresponden con los volcanes Zempoala y La Corona, que conforman la terminación sur de la sierra, con edades de 1.0 a $0.7 \mathrm{Ma}$ (Arce et al., 2008). En la parte media de la Sierra de las Cruces, existen varios fechamientos, que van desde 2.0 hasta $0.7 \mathrm{Ma}$ (Mooser et al., 1974; MoraAlvarez et al., 1991; Osete et al., 2000). De manera general, los productos de estas estructuras volcánicas consisten de derrames de lava, depósitos piroclásticos, depósitos de flujos de escombros y avalanchas de escombros (Aguirre-Díaz et al., 2006; García-Palomo et al., 2008; Arce et al., 2008), con composición química de andesita a dacita de afinidad calci-alcalina (Gunn y Mooser, 1970; Arce et al., 2008).

De manera particular, en el área de estudio se encuentran los volcanes San Miguel y Ajusco, como parte de la Sierra de las Cruces (Figura 1B). El volcán San Miguel o también definido como Complejo Volcánico San Miguel (RomeroTerán, 2001; García-Palomo et al., 2008), consiste de varias estructuras (domos, derrames de lava) amalgamados y afallados (Colín-Rodríguez, 2006; García-Palomo et al., 2008). El volcán San Miguel está conformado por secuencias espesas de derrames de lava, de composición andesítica $\left(61 \%\right.$ en peso de $\mathrm{SiO}_{2}$ ) y dacítica ( $63 \%$ en peso de $\mathrm{SiO}_{2}$ ) (Figura 3; Tabla 1). Las lavas presentan textura porfídica; las andesitas están conformadas por fenocristales de plagioclasa + orto y clinopiroxeno + anfíbol + óxidos de Fe-Ti; mientras que las dacitas por fenocristales de plagioclasa + anfíbol + biotita + cuarzo y en menor cantidad ortopiroxeno + óxidos de Fe-Ti (Figura 5), inmersos en una matriz microlítica y vítrea.

Por otra parte, la denominada Formación Xochitepec fue definida por Mooser et al. (1956) como una serie de rocas volcánicas brechadas y afalladas, de composición andesítica y con una edad del Oligoceno, con afloramientos en el poblado de Santa Cruz Xochitepec, en la Delegación Xochimilco (Figura 6). Posteriormente esta misma 

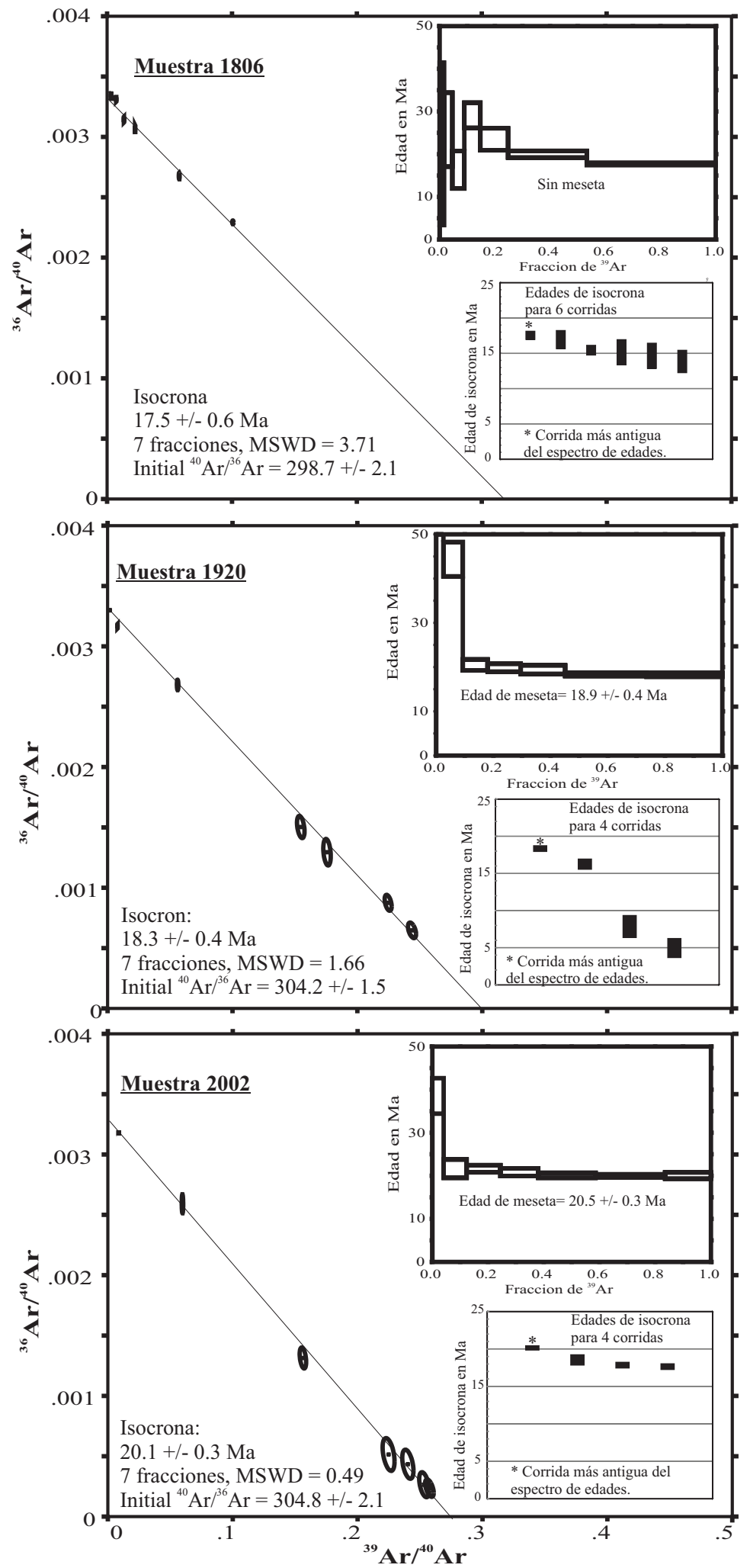

Figura 4. Correlación y espectros de edad de los análisis ${ }^{40} \mathrm{Ar} /{ }^{39} \mathrm{Ar}$, con edades preferidas de fragmentos de muestras individuales, para las tres muestras del pozo profundo San Lorenzo Tezonco. La incertidumbre de las edades se presenta con errores de 1-sigma para cada fragmento. La isócrona y espectro de edad muestra resultados de la corrida más antigua y mejor definida de cada muestra (marcada con * en el recuadro de la figura). Para la muestra 1806, ninguna de las muestras presentó una isócrona verdadera o edad de meseta, reflejando alteración, por lo tanto la edad mejor definida de $17.5 \pm 0.6 \mathrm{Ma}$, probablemente refleja pérdida de argón. Para la muestra 1920, dos de los cuatro fragmentos analizados, arrojaron edades de menos de 10 Ma, reflejando contaminación con material más joven, no identificado durante la selección de las muestras. Sin embargo, la edad más antigua de la muestra 1920 (18.3 \pm $0.4 \mathrm{Ma})$ y la edad de la muestra $2002(20.1 \pm 0.3 \mathrm{Ma})$ muestran una isócrona y edad de meseta bien definida, interpretada como la edad del magmatismo asociado con estas lavas. 

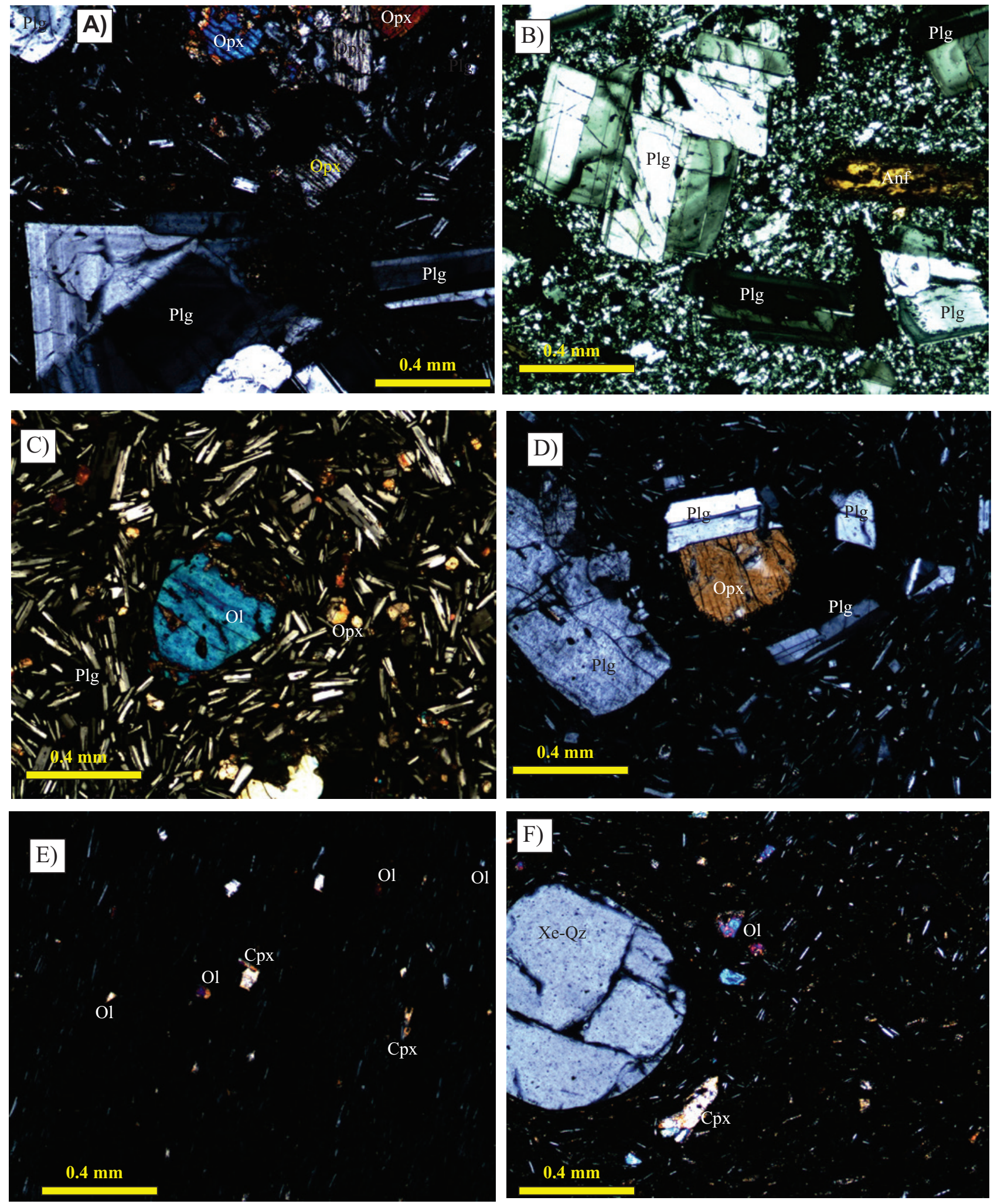

Figura 5. Microfotografías con luz polarizada, de muestras de distintas estructuras volcánicas mencionadas en el texto. A) Dacita del volcán San Miguel; B) Dacita de Xochitepec; C) Dique de andesita basáltica de Xochitepec; D) Andesita de Cerro de la Estrella; E) Andesita basáltica de Santa Catarina; F) Andesita basáltica del volcán Tezontle (Campo Volcánico Chichinautzin). Plg, plagioclasa; Opx, ortopiroxeno; Ol, olivino; Anf, anfíbol; Cpx, clinopiroxeno; Xe-Qz, xenocristal de cuarzo. 
formación fue mencionada por Colín-Rodríguez (2006) como una serie de derrames de lava y brechas volcánicas de composición dacítica y hasta riolítica. Sin embargo, en ninguno de estos dos trabajos se proporcionan tablas con datos de la composición química ni de edades radiométricas.

En el presente trabajo se realizaron descripciones de varios afloramientos del área de Xochitepec. En general las rocas que conforman a la denominada Formación Xochitepec, constan de derrames de lava y brechas (que podría corresponder con autobrechamiento y/o brechas de falla). Además se describió un dique de color gris oscuro, orientado NO-SE, de textura afanítica (en muestra de mano) de composición andesítico-basáltica $(54.8 \%$ en peso de $\mathrm{SiO}_{2}$ ) (Figura 3; Tabla 1) y en lámina delgada presenta fenocristales de plagioclasa + clinopiroxeno + olivino y óxidos de Fe-Ti. Mientras que las lavas de Xochitepec son predominantemente de composición dacítica (64 - 66.7\% en peso de $\mathrm{SiO}_{2}$ ) (Figura 3; Tabla 1) y en menor cantidad andesitas. Lamentablemente no fue posible obtener una muestra sana para poder llevar a cabo análisis químicos de las andesitas. En general la textura de las lavas de Xochitepec es porfídica, con fenocristales, en distintas proporciones, de plagioclasa + piroxeno + biotita + cuarzo + óxidos de FeTi, inmersos en una matriz microlítica y vítrea (Figura 5). Además se llevaron a cabo tres fechamientos radiométricos por el método U-Pb en circones, que sorprendentemente resultaron en edades de 1.23 a 1.66 Ma (Figura 7; Tabla 3), muy contrastantes con la edad del Oligoceno mencionada en trabajos previos (Mooser et al., 1956).

\subsection{Cerro de la Estrella y Sierra Santa Catarina}

Son escasos los trabajos que se han llevado a cabo sobre el Cerro de la Estrella y Sierra Santa Catarina. Comúnmente se trata de trabajos muy generales, como los realizados por Waitz (1910 y 1911) con aspectos meramente descriptivos de las estructuras y tipos de roca (en muestra de mano). Aunque en el trabajo de Lugo-Hubp et al. (1994) se reporta un análisis morfológico detallado de cada estructura volcánica, hasta la fecha no se cuenta con una descripción más detallada de los productos de dicha sierra, ni tampoco datos de la composición química de sus rocas. Mientras que para el Cerro de la Estrella, la información es aún más escasa.

Tal como se ha manejado en trabajos previos (i.e.: Vázquez-Sánchez y Jaimes-Palomera, 1989; Lugo-Hubp et al., 1994), el Cerro de la Estrella es independiente de la Sierra Santa Catarina, debido a que presentan características morfológicas diferentes (Figura 6). El Cerro de la Estrella se define en este trabajo como un "volcán en escudo", con base en sus características morfológicas, ya que presenta pendientes y terminaciones de los frentes de lava muy suaves, en forma radial, coronada por un cono de escorias. Resalta además el grado de erosión que presenta esta

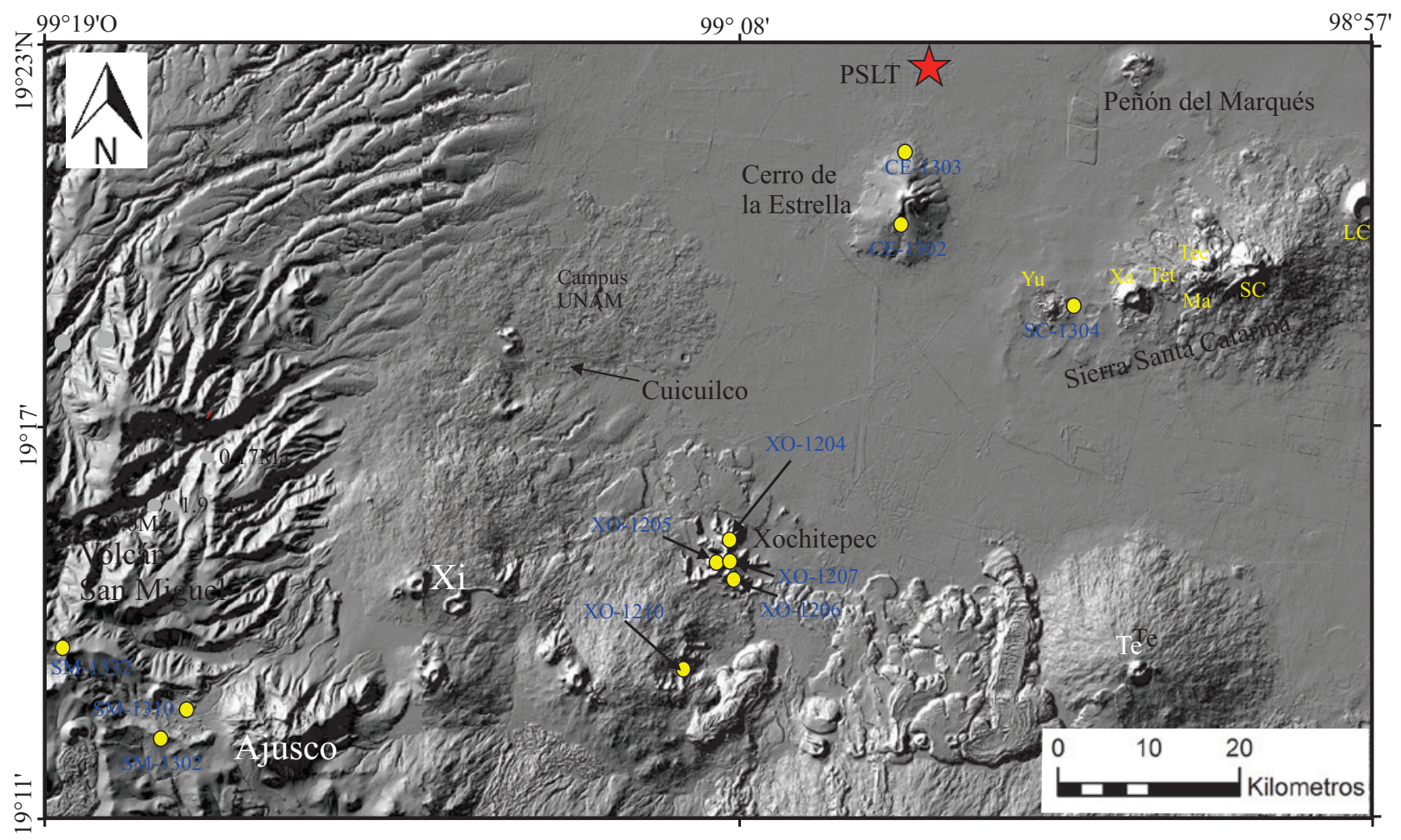

Figura 6. Modelo digital de elevación de la parte sur de la Cuenca de México, en el que se señala la ubicación de las muestras discutidas en este trabajo, así como algunas estructuras volcánicas. Xi, Xitle; Te, Teutli; Yu, Yuhualixqui; Xa, Xaltepec; Tet, Tetecón; Ma, Mazatepec; Tec, Tecuatzi; SC, Santa Catarina; LC, La Caldera y el Pozo San Lorenzo Tezonco, SLT. 

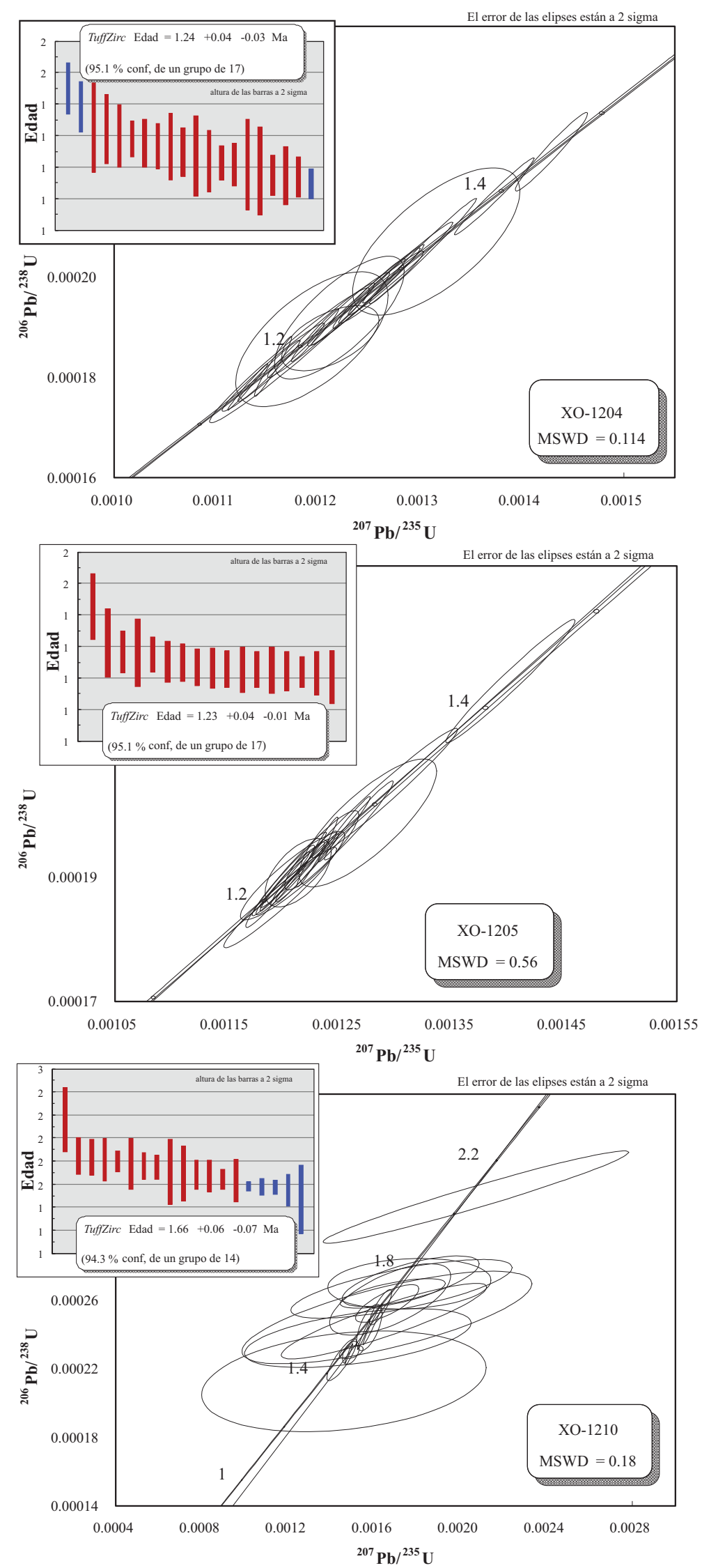

Figura 7. Diagramas de Concordia Tera-Wasserbug U-Pb, considerando los circones representativos de las distintas muestras. Las edades promedio de ${ }^{206} \mathrm{~Pb} /{ }^{238} \mathrm{U}$ de los circones fueron calculadas usando el algoritmo Tuff Zirc de Ludwig y Mundil (2002) (ver Tabla 3). 
estructura (drenaje definido) con respecto a Santa Catarina. Los productos del Cerro de la Estrella (escorias y derrames de lava), presentan una composición andesítica (58\% en peso de $\mathrm{SiO}_{2}$ ) (Figura 3; Tabla 1), con una textura porfídica y fenocristales de plagioclasa + clinopiroxeno + óxidos de Fe-Ti (Figura 5), así como xenocristales de cuarzo. No existe ningún fechamiento radiométrico para esta estructura hasta la fecha.

La Sierra Santa Catarina está conformada de manera general, por siete estructuras volcánicas monogenéticas, alineadas en dirección general E-O, y de occidente a oriente son denominadas Yuhualixqui, Xaltepec, Tetecón, Tecuatzi, Mazatepec, Santa Catarina y La Caldera (Lugo-Hubp et al., 1994). La mayoría se trata de conos de escoria y cenizas, con derrames de lava asociados. Una de estas estructuras consiste de un domo de lava (Tecuatzi) y la estructura más al occidente se trata de un maar (La Caldera) (Lugo-Hubp et al., 1994), con un cráter de dimensiones relativamente grande (1.4 km eje mayor) y de poca altura $(195 \mathrm{~m})$. Actualmente se aprecian bancos de material, en donde es posible hacer descripciones y tomar muestras de los depósitos de escoria, pero para los derrames de lava, existen afloramientos muy restringidos y la mancha urbana hace aún más difícil el acceso a algún afloramiento representativo.

En el presente trabajo, sólo se reporta una muestra que fue tomada del volcán Yuhualixqui, muy cerca del cono de este nombre y corresponde a una bomba volcánica, de composición en el límite andesítico-basáltico y andesítico (57\% en peso de $\mathrm{SiO}_{2}$ ) (Figura 3; Tabla 1). En general la roca es afanítica en muestra de mano, pero bajo el microscopio se aprecia una textura porfídica, con fenocristales de plagioclasa + clinopiroxeno + ortopiroxeno + olivino y xenocristales de cuarzo, inmersos en una matriz vítrea (Figura 5).

La edad para la Sierra de Santa Catarina no ha sido bien establecida, aunque se ha inferido que dicha sierra podría ser más joven que 20 mil años (Lugo-Hubp et al., 1994). Los primeros intentos en determinar la edad absoluta fueron hechos por Layer et al. (2009b), mediante fechamientos radiométricos por el método ${ }^{40} \mathrm{Ar} /{ }^{39} \mathrm{Ar}$, pero los resultados no fueron muy precisos, probablemente debido al tipo de roca (pobre en potasio) y a su juventud, y estos mismos autores proponen que podría tratarse de volcanes del Holoceno.

\subsection{Campo Volcánico Chichinautzin}

El Campo Volcánico Chichinautzin forma parte del límite sur de la Cuenca de México (Figura 1B), constituido por más de 120 volcanes monogenéticos (conos de escoria, derrames de lava fisurales, domos y volcanes en escudo), dentro de un área de $2680 \mathrm{~km}^{2}$, formando una banda irregular alargada en dirección E-O, desde las faldas del volcán Popocatépetl hasta las faldas del Nevado de Toluca (Bloomfield, 1975; Martin del Pozzo, 1982; Marquez et al., 1999; Siebe et al., 2004).

Este campo volcánico es muy peculiar, debido a que sus productos tienen una composición heterogénea y por lo mismo, ha habido muchos estudios y discusiones enfocados a entender la génesis de los magmas primarios y su evolución (Wallace y Carmichael, 1999; Márquez et al., 1999; Verma, 2000; Sheth et al., 2002; Meriggi et al., 2008; Straub et al., 2011; 2013). De este modo, existe una gran cantidad de datos, desde análisis petrográficos hasta datos de relaciones isotópicas y hasta la fecha aún siguen en discusión varios aspectos del vulcanismo de este campo. Sin embargo, el objetivo de este artículo es solamente plantear las características particulares de dicho campo volcánico, con el fin de poder realizar correlaciones con otras unidades litológicas.

Para el presente trabajo solamente se presenta un análisis químico de roca total y corresponde con el cono monogenético Tezontle, localizado al SO del volcán San Miguel (Figura 6; muestra SM-1310). El volcán Tezontle tiene una composición química andesítico-basáltica (57 $\%$ en peso de $\mathrm{SiO}_{2}$ ) (Figura 3; Tabla 1) y corresponde con la composición más común del Campo Volcánico Chichinautzin (e.g.: Wallace y Carmichael, 1999). El intervalo de edades para las estructuras volcánicas de Chichinautzin es muy amplio, desde 1.2 Ma (Arce et al., 2013b), hasta 1600 años A.P., (Siebe, 2000), este último corresponde con la edad del volcán Xitle (Figura 6), con extensos derrames de lava basáltica emplazados hacia el norte del volcán, alcanzando la zona donde actualmente se encuentra la Ciudad Universitaria de la UNAM.

\section{Discusión}

\subsection{Relaciones estratigráficas}

En el presente trabajo se aportan datos radiométricos obtenidos con los métodos ${ }^{40} \mathrm{Ar} /{ }^{39} \mathrm{Ar}$ y U-Pb en circones (Tablas 2 y 3), relaciones de campo, así como descripciones petrográficas y análisis químicos de roca total (Tabla 1). Con estas herramientas se intentó llevar a cabo una correlación de las unidades litológicas descritas en el pozo San Lorenzo Tezonco, con la geología superficial.

Como se aprecia en la columna litológica del pozo profundo (Figura 2), se proponen cuatro grandes paquetes litológicos. Desde el más profundo al más somero, se tiene al paquete de la Formación Tepoztlán, siendo el más importante en espesor en el pozo profundo, constituido por una gran variedad de litologías, abarcando desde los 876 hasta los 2008 metros de profundidad (Figura 2). Las razones por las que se propone su correlación con las rocas de la Formación Tepoztlán, que aflora en dos zonas principales (Tepoztlán, Morelos y Malinalco, Estado de México), se basan en su composición química variable, desde andesitas basálticas hasta dacitas (Figura 3) e incluso riolitas (Lenhardt, 2009). Desafortunadamente no se han reportado datos químicos completos (elementos mayores y trazas) por lo tanto no es posible hacer más 
comparaciones químicas. Sin embargo, se ha determinado que la Formación Tepoztlán está conformada por derrames de lava, intercalados con depósitos piroclásticos y lahares, similar a su correspondiente en el pozo profundo. Un elemento adicional para proponer esta correlación es la edad, ya que a una profundidad de $\sim 965 \mathrm{~m}$, se determinó una edad de $13.5 \mathrm{Ma}$, a $\sim 1286 \mathrm{~m}$ una edad de $17 \mathrm{Ma}, \mathrm{a} \sim 1550$ una edad de $18.4 \mathrm{Ma}$; y los nuevos fechamientos ${ }^{40} \mathrm{Ar} /{ }^{39} \mathrm{Ar}$ oscilan entre $18 \mathrm{Ma}(1820 \mathrm{~m})$ hasta $20.1 \mathrm{Ma}(2002 \mathrm{~m}$ de profundidad) (Figura 4), que concuerda con el intervalo de edades propuesto para la Formación Tepoztlán entre 11 y 22.8 Ma (Lenhardt et al., 2010).

Posteriormente se tiene al grupo de rocas comprendidas entre las profundidades de 581 a 875 metros, que en el presente trabajo se le correlaciona con rocas de la Sierra de las Cruces. La litología descrita en el pozo profundo varía de lavas de composición dacítica a depósitos piroclásticos de composición riolítica (Arce et al., 2013a), con edades de 0.9 a 5 Ma. A pesar de que en superficie, las edades más antiguas para la Sierra de las Cruces van desde los 2 hasta 3.7 Ma (Mora-Alvarez et al., 1991; Osete et al., 2000; Aguirre-Díaz et al., 2006), no se descarta la posibilidad de que existan productos más antiguos similares a las rocas de 5 Ma descritas en el pozo, pero que probablemente han quedado sepultadas por productos más recientes, o que no han sido reconocidas en el campo. Un elemento importante para esta correlación, además de la edad, es la composición química (Figuras 3 y 8) y la textura porfídica de grano grueso de ambos grupos litológicos (muestras superficiales y muestras del pozo profundo). Es de notar que en casi todos los diagramas binarios (Figura 8) la composición química de ambos es muy parecida, al igual que las muestras de Xochitepec. Los elementos traza no son la excepción (Figura 9), ya que el patrón que presentan ambos grupos es muy parecido, incluso con las muestras de Xochitepec de nueva cuenta, con anomalías negativas en $\mathrm{Nb}, \mathrm{Ta}, \mathrm{P}, \mathrm{La}$, $\mathrm{Ce}$ y positiva en $\mathrm{Pb}$. Estas características apuntan a rocas generadas en un ambiente de subducción, como el que ocurre en la Trinchera Mesoamericana.

De acuerdo con estas similitudes químicas y complementado con las edades U-Pb en circones entre 1.23 y 1.66 Ma (Figura 7; Tabla 3), se propone que las rocas de Xochitepec son correlacionables con rocas de la Sierra de las Cruces, tanto en superficie como para el pozo profundo.

Posteriormente, en el pozo se tiene a un grupo de rocas de composición andesítica y andesítico-basáltica (Figura 3), entre las profundidades de 70 a 580 metros. Estas lavas han sido correlacionadas con productos del Campo Volcánico Chichinautzin (Arce et al., 2013a). Sin embargo, con base en la cercanía (Figura 6), edades propuestas y composición química, en el presente trabajo se correlaciona a estas rocas con el Cerro de la Estrella (andesitas de 0.25 Ma en el pozo) y Sierra Santa Catarina (andesitas basálticas). En el presente trabajo se reportan datos químicos del Cerro de la Estrella y Santa Catarina que afloran en la superficie (Tabla 1) y como se aprecia en los diagramas binarios (Figura 8) y diagramas de multielementos (Figura 9), son muy parecidos a las rocas descritas en el pozo profundo, en los intervalos mencionados. Se destaca el enriquecimiento relativo en $\mathrm{P}_{2} \mathrm{O}_{5}, \mathrm{MgO}, \mathrm{CaO}, \mathrm{Fe}_{2} \mathrm{O}_{3} \mathrm{TiO}_{2}$ (Figura 8), así como las anomalías menos pronunciadas en $\mathrm{Nb}$, Ta, La y Ce (Figura 9) con respecto a las demás muestras. Además, los patrones de tierras raras son muy similares para las muestras de Cerro de la Estrella, Santa Catarina y sus correspondientes en el pozo profundo. Adicionalmente llama la atención el dique descrito en las rocas de Xochitepec (muestra XO-1207; Tabla 1), el cual tiene una composición andesítico-basáltica (Figura 3), con concentraciones en elementos mayores (Figura 8) y patrones en los diagramas multielementos y tierras raras (Figura 9) muy similares a las rocas de Santa Catarina y Cerro de la Estrella. Es probable que el dique corresponda con algún evento asociado a estas estructuras volcánicas.

Por último, se tiene al paquete más superficial, el cual consiste de depósitos lacustres, con edades tan antiguas de más de 220 mil años A.P., (Brown et al., 2012) hasta el Reciente. La sedimentación en el lago que albergaba la Cuenca de México, se vio interrumpida en varias ocasiones por eventos volcánicos como el de la Pómez Tutti Frutti hace 14000 años (Siebe y Macías, 2004), Pómez Toluca Superior hace 10500 años (Bloomfield et al., 1977; Arce et al., 2003) y el volcán Xitle hace 1600 años (Siebe, 2000).

\subsection{Geología actualizada del área}

Con base en los resultados que se han presentado en este trabajo, complementado con información de trabajos previos, se elaboró un mapa de la distribución de las distintas rocas que corresponden a las estructuras que se encuentran en la parte sur de la Cuenca de México (Figura 10). Las rocas más antiguas en esta zona, corresponden con los productos de los volcanes San Miguel y Ajusco, ambos pertenecientes a la Sierra de las Cruces. Las edades que se han reportado para ambas estructuras oscilan entre 1.9 y $0.4 \mathrm{Ma}$ (MoraAlvarez et al., 1991; Osete et al., 2000; Mejia et al., 2005), con composición variable de andesita a dacita. De manera general, estos volcanes poligenéticos están conformados por derrames de lava, domos, depósitos piroclásticos y lahares, de composición andesítica y dacítica (RomeroTerán, 2001; Arce et al., 2008). En la parte noreste de San Miguel se aprecian abanicos, con drenaje bastante profundo, los cuales están conformados por depósitos piroclásticos (flujos piroclásticos y pómez de caída) y depósitos de flujos de escombros. Hacia el este de San Miguel se observa en el mapa una serie de cuerpos aislados (cerro Zacatépetl, Parque Bosque de Tlalpan, entre otros), con una morfología muy particular, contrastante con los derrames de lava del Xitle (Figura 10). Es probable que estos cuerpos aislados sean remanentes del volcán San Miguel, debido a su cercanía, aunque tampoco se puede descartar que estén asociados con el volcán Ajusco como ha sido propuesto (Cervantes y Molinero, 1995). Los cuerpos aislados están constituidos 


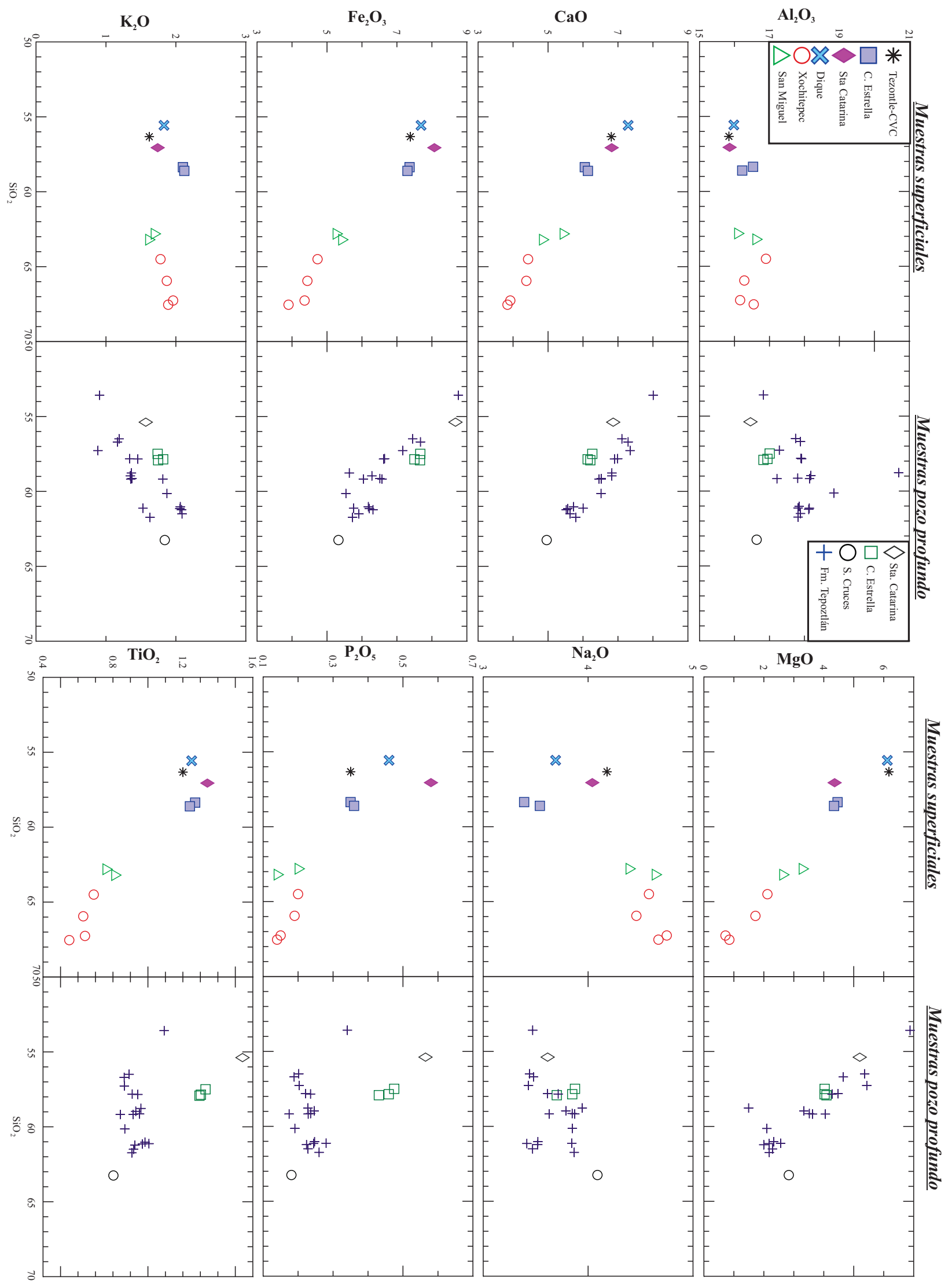

Figura 8. Diagramas binarios $\mathrm{SiO}_{2}$ vs. elementos mayores de muestras del pozo San Lorenzo Tezonco y muestras de volcanes que afloran en superficie (ver Tabla 1). Fe total reportado como $\mathrm{Fe}_{2} \mathrm{O}_{3}$. Todos los datos fueron normalizados en base anhidra. Las muestras del pozo profundo fueron tomadas de Arce et al. (2013a). 

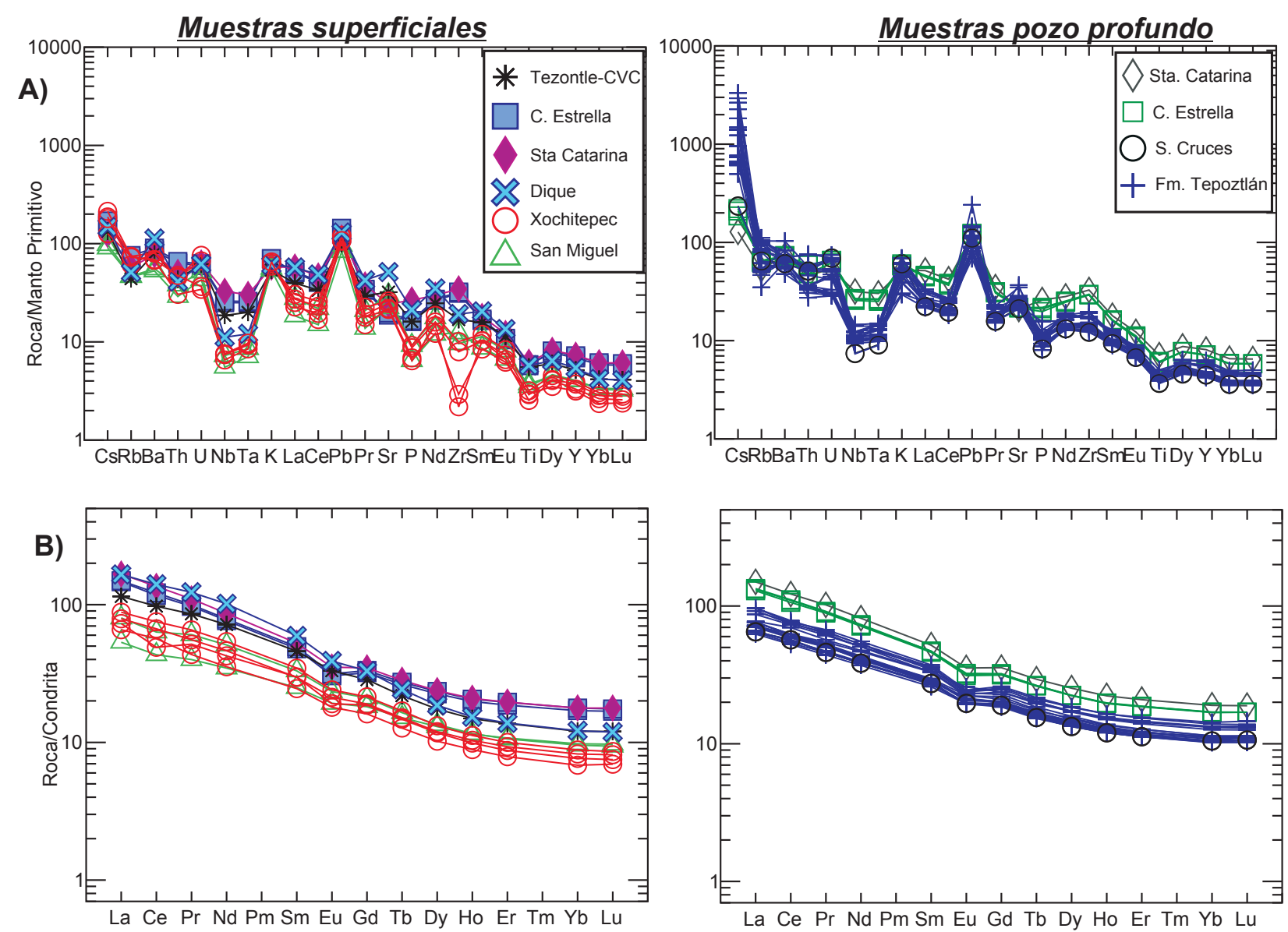

Figura 9. Diagramas del contenido de elementos traza, A) normalizados con el manto primitivo (Sun y McDonough, 1989) y B) datos de tierras raras normalizados con valores de la Condrita (Sun y McDonough, 1989). Los datos del pozo fueron tomados de Arce et al. (2013a) y los datos de muestras de estructuras volcánicas superficiales son de este trabajo.

por depósitos de flujos de escombros endurecidos, con algunas estructuras en rompecabezas (típico en avalanchas de escombros), pero también se han reconocido depósitos de pómez de caída de composición dacítica.

De acuerdo con las edades de las rocas de Xochitepec, definitivamente no son del Oligoceno, por el contrario coinciden con edades y composiciones de la Sierra de las Cruces. Con base en estos resultados es muy posible que dicha formación más bien sea parte de la Sierra de las Cruces (Figura 6), pero que debido a la actividad reciente del Campo Volcánico Chichinautzin, así como a la tectónica de esta región (García-Palomo et al., 2008; Arce et al., 2013a), estos cuerpos volcánicos (Xochitepec, cerro Zacatépetl, etc.) quedaron parcialmente cubiertos por lavas del Xitle y otros volcanes monogenéticos del Chichinautzin.

El Cerro de la Estrella tiene una forma semicircular, constituido por lavas, con pendientes suaves y coronado por un cono de escoria, clasificado en este trabajo como un volcán escudo. Se logra apreciar el drenaje natural hacia la pendiente noreste del volcán, por lo que se deduce que es relativamente más antiguo que la Sierra Santa Catarina, pero contemporáneo con el volcán monogenético Peñón del Marqués (Figura 10), basándose solamente en la degradación de ambos. La Sierra Santa Catarina por el contrario, presenta una morfología bastante bien definida, lo que sugiere una edad muy joven, probablemente del Holoceno (Layer et al., 2009b).

En términos estrictos, es muy difícil delimitar (espacial y temporalmente) al Campo Volcánico Chichinautzin, ya que su intervalo composicional es muy amplio (aunque con predominio de andesitas basálticas) y con edades entre 1.2 Ma hasta 1600 años A.P., (Siebe, 2000; Arce et al., 2013b). En este sentido, el vulcanismo de la Sierra Santa Catarina bien podría corresponder con el vulcanismo en Chichinautzin, con base en la posible edad holocénica y la composición química (Tabla 1).

Finalmente, la parte sur del mapa de la Figura 10, lo conforma la parte norte del Campo Volcánico Chichinautzin, con varias estructuras monogenéticas, de las cuales resalta el volcán escudo Teutli (Figura 10) con una edad de $>14$ mil años A.P. (Siebe et al., 2005), también se aprecian derrames de lava muy viscosos, aparentemente fisurales, asociados a la Falla Xochimilco (García-Palomo et al., 2008) y el Xitle de 1600 años A.P. (Siebe, 2000). 


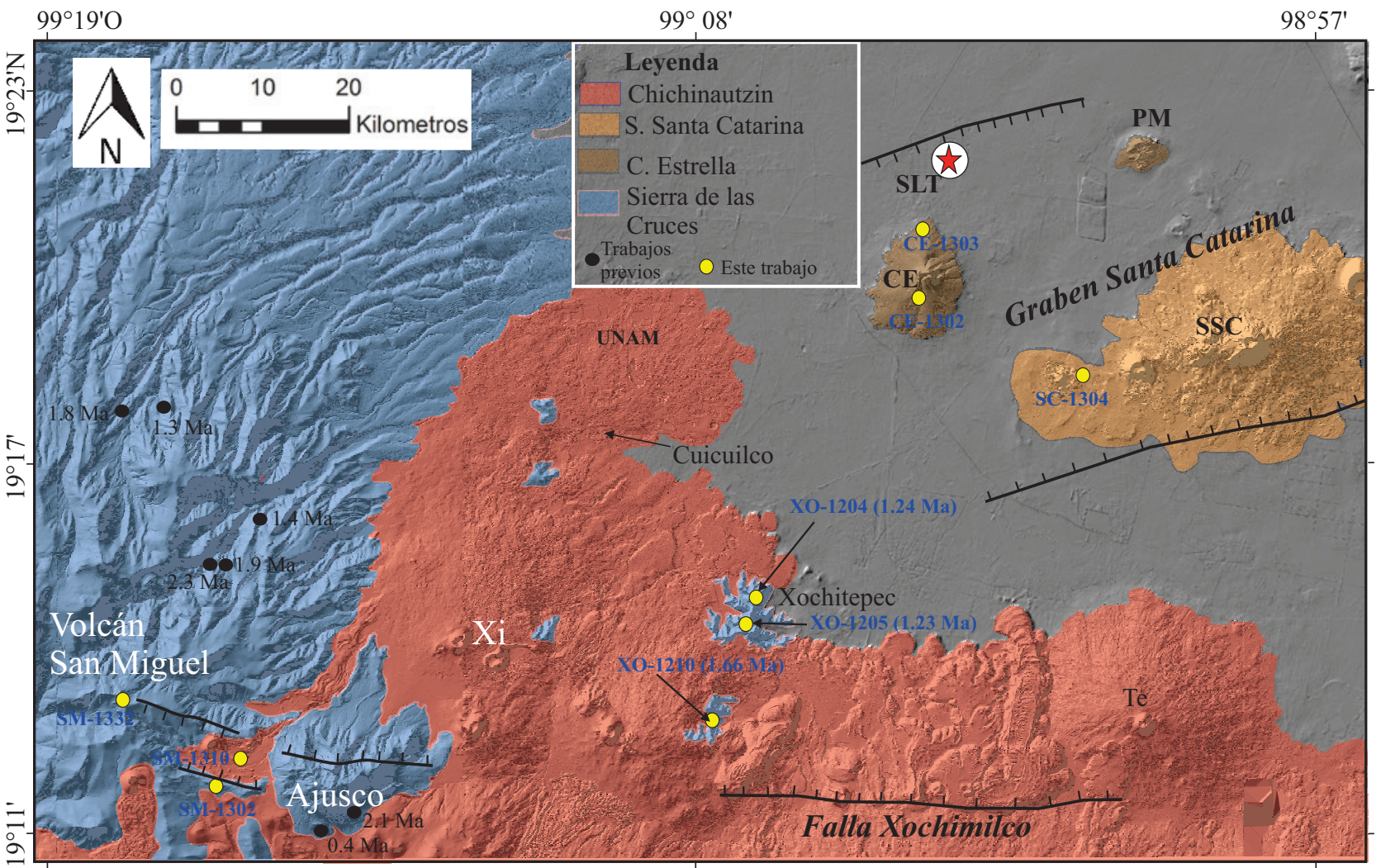

Figura 10. Mapa de la distribución de las distintas unidades litológicas discutidas en el texto. Se muestran los afloramientos mencionados así como las edades obtenidas por el método U-Pb en circones de las rocas de Xochitepec. Las edades de los círculos negros fueron tomados de Mejia et al. (2005); Mora-Alvarez et al. (1991); Osete et al. (2000) Romero-Terán (2001). CE, Cerro de la Estrella; SSC, Sierra Santa Catrina; PM, Peñón del Marqués; PSLT, Pozo San Lorenzo Tezonco. La falla Xochimilco y las fallas en los volcanes San Miguel y Ajusco fueron propuestos por García-Palomo et al. (2008) y las fallas del graben de Santa Catarina fue retomado de Arce et al. (2013a).

\section{Conclusiones}

Las conclusiones que se derivan del presente estudio son los siguientes:

1. A pesar del tipo de muestreo que se llevó a cabo en el pozo San Lorenzo Tezonco, fue posible dividir en cuatro paquetes litológicos distintos a toda la secuencia volcánica y lacustre del pozo.

2. Los paquetes descritos en el pozo profundo fueron correlacionados con estructuras y/o rocas que afloran en los alrededores de la parte sur de la Cuenca de México. Así mismo, al vulcanismo asociado con la Formación Tepoztlán quedó registrado en el pozo como una secuencia volcánica de gran espesor, cuyas edades entre 13 y 20.1 Ma coinciden perfectamente con el intervalo de edad propuesto para esta formación de $11-22.8 \mathrm{Ma}$ (Lenhardt et al., 2010), en los sitios de Tepoztlán, Morelos y Malinalco, Estado de México.

3. Con base en la composición química y edades radiométricas, fue posible determinar que rocas que se habían denominado como Formación Xochitepec (Mooser et al., 1956) del Oligoceno, resultaron ser mucho más jóvenes (1.23 a $1.66 \mathrm{Ma})$ y más bien corresponden con rocas de la Sierra de las Cruces.

4. Además, los remanentes que se observan en el mapa geológico (Figura 10), como el cerro Zacatépetl, Bosque de Tlalpan y otros, es muy probable que estén asociados al volcán San Miguel, pero que movimientos tectónicos posteriores, generaron depresiones que fueron rellenadas por las lavas del Xitle, hace 1600 años y solamente quedaron algunos altos topográficos, como remanentes.

5. Es muy probable que los volcanes monogenéticos de la Sierra Santa Catarina sean del Holoceno (Layer et al., 2009b), y por lo tanto contemporáneos con un periodo de vulcanismo del Campo Volcánico Chichinautzin. Además, la composición química de ambos es muy similar. Mientras que el Cerro de la Estrella es un poco más antiguo, tal vez equivalente a la andesita fechada en $0.25 \mathrm{Ma}$ en el pozo profundo (Arce et al., 2013a).

\section{Agradecimientos}

Un especial agradecimiento al Sistema de Aguas de la Ciudad de México por proporcionar las muestras de canal, utilizadas en los fechamientos radiométricos de ${ }^{40} \mathrm{Ar} r{ }^{39} \mathrm{Ar}$. 
Los análisis ${ }^{40} \mathrm{Ar} /{ }^{39} \mathrm{Ar}$ se realizaron en el Laboratorio de Geocronología de la Universidad de Alaska, Fairbanks, E.U.A. Los fechamientos por el método U-Pb en circones fueron realizados en el Centro de Geociencias, UNAM por L. Solari y C. Ortega-Obregón. Los análisis químicos de roca total se llevaron a cabo en el LUGIS, UNAM por P. Girón y los análisis por ICP-MS se realizaron en el Centro de Geociencias, UNAM, Juriquilla, Qro., por O. Pérez-Arvizu. Esta investigación ha sido financiada por los proyectos PAPIIT-IN104214-3 y CONACYT 98772 (J.L. Arce). Algunos resultados presentados en este trabajo forman parte de los proyectos de tesis de los estudiantes J.I. Salinas (Facultad de Ingeniería, UNAM) e I. Martínez (ESIA-IPN). Gracias a G. Zenteno por su apoyo en las salidas de campo en el área de Xochitepec. Los comentarios de dos revisores anónimos ayudaron a mejorar de manera importante el manuscrito original.

\section{Referencias}

Aguirre-Díaz, G.J., López-Martínez, M., Rendón-Márquez, G., 2006, La Caldera de La Catedral, Sierra de Las Cruces, una caldera al norponiente de la Ciudad de México (resumen), Reunión Anual de la Unión Geofísica Mexicana, Puerto Vallarta, Jalisco, México: GEOS, 26, 160-161.

Agustín-Flores, J., Siebe, C., Guilbaud, M.N., 2011, Geology and geochemistry of Pelagatos, Cerro del Agua, and Dos Cerros monogenetic volcanoes in the Sierra Chichinautzin Volcanic Field, south of México City: Journal of Volcanology and Geothermal Research, 201, 143-162.

Arce, J.L., Macías, J.L., Vázquez-Selem, L., 2003, The 10.5 ka Plinian eruption of Nevado de Toluca volcano, Mexico: Stratigraphy and hazard implications: Geological Society of America Bulletin, 115, 230-248.

Arce, J.L., Macías, R., García-Palomo, A., Capra, L., Macías, J.L., Layer, P., Rueda, H., 2008, Late Pleistocene flank collapse of Zempoala Volcano (Central Mexico) and the role of fault reactivation: Journal of Volcanology and Geothermal Research, 177, 944-958.

Arce, J.L., Layer, P.W., Morales-Casique, E., J., Benowitz, J.A:, Rangel, E., Escolero, O., 2013a, New constraints on the subsurface geology of the Mexico City Basin: The San Lorenzo Tezonco deep well, on the basis of ${ }^{40} \mathrm{Ar} /{ }^{39} \mathrm{Ar}$ geochronology and whole-rock chemistry: Journal of Volcanology and Geothermal Research, 266, 34-49.

Arce, J.L., Layer, P.W., Lassiter, J., Benowitz, J.A., Macías, J.L., RamírezEspinosa, J., 2013b, ${ }^{40} \mathrm{Ar} /{ }^{39} \mathrm{Ar}$ dating, geochemistry, and isotopic analyses of the Quaternary Chichinautzin Volcanic Field, south of Mexico City: Implications for timing, effusion rate, and distribution of the volcanism: Bulletin of Volcanology, 75:774, doi: 10.1007/ s00445-013-0774-6.

Bloomfield, K., 1975, A late-Quaternary monogenetic volcano field in central Mexico: Geologische Rundschau, 64, 476-497.

Bloomfield, K., Sánchez-Rubi, G., Wilson, L., 1977, Plinian Eruptions of Nevado de Toluca: Geologische Rundschau, 66, 120-146.

Brown, E.T., Werne, J.P., Lozano-García, M.S., Caballero-Miranda, M., Ortega-Guerrero, B., Cabral-Cano, E., Valero-Garcés, B.L., Schwalb, A., Arciniega-Ceballos, A., 2012, Worshop Reports, Scientific Drilling No. 14, 72-75.

Caballero, M.M., Ortega-Guerrero, B., 1998, Lake levels since 40000 ago at Chalco Lake, near Mexico City: Quaternary Research, 50, 90-106.

Cadoux, A., Missenard, Y., Martínez-Serrano, R., Guillou, H., 2011, Trenchward Plio-Quaternary volcanism migration in the TransMexican Volcanic Belt: the case of the Sierra Nevada range: Geological Magazine, doi:10.1017/S0016756810000993.

Cervantes, P., Molinero, R., 1995, Eventos volcánicos al sur de la ciudad de
México: Ciudad de México, D.F., Universidad Nacional Autónoma de México, tesis de licenciatura, $74 \mathrm{p}$.

Colín-Rodríguez, A., 2006, Neotectónica de la Semifosa TláhuacTulyehualco: México D.F., Instituto Politécnico Nacional, Escuela Superior de Ingeniería y Arquitectura, tesis de licenciatura, $75 \mathrm{p}$.

De Cserna, Z., Aranda-Gómez, J.J., Mitre-Salazar, L.M., 1988, Estructura geológica, gravimetría, sismicidad y relaciones neotectónicas regionales de la cuenca de México: Boletín del Instituto de Geología, UNAM, México, 104, 1-71.

Enciso-de la Vega, S., 1992, Propuesta de nomenclatura estratigráfica para la cuenca de México: Universidad Nacional Autónoma de México, Instituto de Geología, Revista, 10, 26-36.

Ferrari, L.M., López-Martínez, G., Aguirre-Díaz, G., Carrasco-Nuñez, G., 1999, Space-time patterns of Cenozoic arc volcanism in central Mexico: From Sierra Madre Occidental to the Mexican Volcanic Belt: Geology, 27, 303-306.

Fries, C., 1956, Bosquejo Geológico de la región entre México, D.F. y Taxco Guerrero, en Congreso Geológico Internacional 20, México, D.F. Libreto de las excursiones Excursión A-9 y C-12,11.

Fries, C., 1960, Geología del Estado de Morelos y de partes adyacentes de México y Guerrero: Región central meridional de México: Boletín del Instituto de Geología, UNAM 60, 234 p.

García-Palomo, A., Zamorano, J.J., López-Miguel, C., Galván-García, A., Carlos-Valerio, V., Ortega, R., Macías, J.L., 2002, El arreglo morfoestructural de la Sierra de las Cruces, México central: Revista Mexicana de Ciencias Geológicas, 25, 158-178.

García-Palomo, A., Macías, J.L., Tolson, G., Valdez, G., Mora, J.C., 2008, Volcanic stratigraphy and geological evolution of the Apan región, east-central sector of the Trans-Mexican Volcanic Belt: Geofisica Internacional, 41, 133-150.

Gunn, B.M., Mooser, F., 1970, Geochemistry of the volcanics of Central Mexico: Bulletin of Volcanology, 34, 577-616.

Layer, P.W., 2000, ${ }^{40} \mathrm{Ar} /{ }^{39} \mathrm{Ar}$ age of the El'gygytgyn impact event, Chukotka, Russia: Meteoritic and Planetary Science, 35, 591-599.

Layer, P.W., Garcia-Palomo, A., Jones, D., Macias, J.L., Arce, J.L., Mora, J.C., 2009a, El Chichon volcanic complex, Chiapas, Mexico: Stages of evolution based on field mapping and ${ }^{40} \mathrm{Ar} /{ }^{39} \mathrm{Ar}$ geochronology: Geofisica Internacional, 48, 33-54.

Layer, P.W., Macias, J.L., Arce, J.L., García-Tenorio, F., 2009b, Late Pleistocene-Holocene volcanism of the Mexico Basin and assessment of volcanic hazards in one of the world's largest cities: American Geophysical Union, Fall Meeting, 1, 1951 p.

Le Bas, M.J., Le Maitre, R.W., Streckaisen, A., Zanetti, B., 1986, A chemical classification of volcanic rocks based on the total alkalisilica diagram: Journal of Petrology, 27, 745-750.

Lenhardt, N., 2009, Volcaniclastic successions of the southern edge of the Transmexican Volcanic Belt: Evidence for the Miocene plate reorganisation in Central America (Morelos, Mexico): Alemania, Universidad Tecnológica de Darmstadt, Tesis de Doctorado, 141 p., http://tuprints.ulb.tu-darmstadt.de/1405/.

Lenhardt, N., Böhnel, H., Wemmer, K., Torres-Alvarado, I.S., Hornung, J., Hinderer, M., 2010, Petrology, magnetostratigraphy and geochronology of the Miocene volcaniclastic Tepoztán Formation: implications for the initiation of the Transmexican Volcanic Belt (Central Mexico): Bulletin of Volcanology, 72, 817-832.

López-Hernández, A., 2009, Evolución volcánica del Complejo Tulancingo-Acoculco y su sistema hidrotermal, estados de Hidalgo y Puebla, México: México, D.F., Universidad Nacional Autónoma de México, tesis de doctorado, $170 \mathrm{p}$.

Lozano-García, M.S., 1989, Palinología y paleoambientes pleistocénicos de la Cuenca de México: Geofísica Internacional, 28, 335-362.

Lozano-García, M.S., Ortega-Guerrero, B., 1998, Late Quaternary environmental changes of the central part of the Basin of Mexico: Correlation between Texcoco and Chalco basins: Review of Palaeobotany and Palynology, 99, 77-93.

Lozano-García, M.S., Ortega-Guerrero, B., Caballero-Miranda, M., Urrutia-Fucugauchi, J., 1993, Late Pleistocene and Holocene paleoenvironments of Chalco Lake, central Mexico: Quaternary Research, 40, 332-342. 
Lozano-Santacruz, R., Bernal, J.P., 2005, Characterization of a new set of eight geochemical reference materials for XRF major and trace element analysis: Revista Mexicana de Ciencias Geológicas, 22, 329-344.

Ludwig, K.L., Mundil, R., 2002, Extracting reliable U-Pb ages and errors from complex populations of zircons from Phanerozoic tuffs, in Paper Presented at J. Conf. Abstr. 12 ${ }^{\text {th }}$ Goldschmidt Conf., 18-23.

Lugo-Hubp, J., Mooser, F., Pérez-Vega, A., Zamorano-Orozco, J., 1994, Geomorfología de la Sierra de Santa Catarina, D.F., México: Revista Mexicana de Ciencias Geológicas, 11, 43-52.

Macdonald, G.A., Katsura, T., 1964, Chemical composition of Hawaiian lavas: Journal of Petrology, 5, 82-133.

MacDonough, W.F., Sun, S.S., 1995, The composition of the earth: Chemical Geology, 120, 223-253.

Macias, J.L., Arce, J.L., García-Tenorio, F., Layer, P.W., Rueda, H., ReyesAgustin, G., López-Pizaña, F., Avellán, D., 2012, Geology and geochronology of Tlaloc, Telapón, Iztaccíhuatl, and Popocatépetl volcanoes, Sierra Nevada, central Mexico, en Aranda-Gómez, J.J., Tolson, G., Molina-Garza, R.S. (Eds.), The Southern Cordillera and Beyond: Geological Society of America Field Guide, 25,163-193.

Márquez, A., Oyarzu, R., Doblas, M., Verma, S.P., 1999, Alcalic (oceanisland basalt type) and calc-alkaline volcanism in the Mexican Volcanic Belt: A case for plume-related magmatism and propagating rifting at an active margin?: Geology, 27, 51-54.

Martin Del Pozzo, A.L., 1982, Monogenetic volcanism in Sierra Chichinautzin, Mexico: Bulletin of Volcanology, 45, 9-24.

McDougall, I., Harrison, T.M., 1999, Geochronology and Thermochronology by the ${ }^{40} \mathrm{Ar} /{ }^{39} \mathrm{Ar}$ method-2nd ed: Oxford University Press, New York, 269 p.

Mejia, V., Böhnel, H., Opdyke, N.D., Ortega-Rivera, M.A., Lee, J.K.W., Aranda-Gómez, J.J., 2005, Paleosecular variation and time-averaged field recorded in late Pliocene-Holocene lava flows from Mexico: Geochemistry Geophysics Geosystems, 6, 1-19.

Meriggi, L., Macías, J.L., Tommasini, S., Capra, L., Conticelli, S., 2008, Heterogeneous magmas of the Quaternary Sierra Chichinautzin volcanic field (central Mexico): the role of an amphibole-bearing mantle and magmatic evolution processes: Revista Mexicana de Ciencias Geológicas, 25, 197-216.

Mooser, F., 1963, Historia tectónica de la Cuenca de México: Boletín de la Asociación Mexicana de Geólogos Petroleros, 15, 239-245.

Mooser, F., 1975, Historia geológica de la Cuenca de México, en Memoria de las obras de drenaje profundo del Distrito Federal, México, D.F., Departamento del Distrito Federal, 38 p.

Mooser, F., White S.E., Lorenzo, J.L., 1956, La Cuenca de México. Consideraciones Geológicas y arqueológicas: México, D.F., Instituto Nacional de Antropología e Historia, 51 p.

Mooser, F., Nair, A.E.M., Negendank, J.F.W., 1974, Paleomagnetic in-vestigations of Tertiary and Quaternary igneous rocks; VII, A paleomegnetic and petrologic study of volcanics of the Valley of Mexico: Geologische Rundschau, 63, 451-483.

Mora-Alvarez, G., Caballero, C., Urrutia-Fucugauchi, J., Uchiumi, S., 1991, Southward migration of volcanic activity in the Sierra de las Cruces, Basin of Mexico. A preliminary K-Ar dating and paleomagnetic study: Geofisica Internacional, 30, 61-70.

Morales-Casique, E., Escolero, O., Arce, J.L., 2014, Resultados del pozo San Lorenzo Tezonco y sus implicaciones en el entendimiento de la hidrogeología regional de la cuenca de México: Revista Mexicana de Ciencias Geológicas, 31, 64-75.

Mori, L., Gómez-Tuena, A., Cai, Y., Goldstein, S.L., 2007, Effects of prolonged flatsubduction on the Miocene magmatic record of the central Trans-Mexican VolcanicBelt: Chemical Geology, 244, $452-473$.

Osete, M.L., Ruíz-Martínez, V.C., Caballero, M.C., Galindo, C., UrrutiaFucugauchi, J., Tarling, H.D., 2000, Southward migration of continental volcanic activity in the Sierra de Las Cruces, Mexico: Paleomagnetic and radiometric evidence: Tectonophysics, 318, 201-215.

Pardo, M., Suárez, G., 1995, Shape of the subducted Rivera and Cocos plates in southern Mexico: seismic and tectonic implications: Journal of Geophysical Research, 100, 12357-12373.

Paton, C., Woodhead, J.D., Hellstrom, J.C., Hergt, J.M., Greig, A., Maas, R., 2010, Improved laser ablation U-Pb zircon geochronology through robust downhole fractionation correction: Geochemistry Geophysics Geosystems, 11, Q0AA06 p, doi: 10.1029/2009GC002618.

Pérez-Cruz, G.A., 1988, Estudio sismológico de reflexión del subsuelo de la Ciudad de México: México D.F., Universidad Nacional Autónoma de México, Faculta de Ingeniería, Tesis de Maestría, 83 p.

Petrus, J.A., Kamber, B.S., 2012, VizualAge: A novel approach to laser ablation ICP-MS U-Pb geochronology data reduction: Geostandards and Geoanalyical Research, 36, 247-270.

Renne, P.R., Mundil, R., Balco, G., Min, K., Ludwig, K., 2010, Joint determination of ${ }^{40} \mathrm{~K}$ decay constants and the ${ }^{40} \mathrm{Ar} * /{ }^{40} \mathrm{~K}$ for the Fish Canyon sanidine standard and improved accuracy for ${ }^{40} \mathrm{Ar} /{ }^{39} \mathrm{Ar}$ geochronology: Geochimica et Cosmochimica Acta, 74, 5349-5367.

Romero-Terán, E., 2001, Geología del Complejo Volcánico San Miguel, al sur poniente de la Cuenca de México, Tesis de Maestría, UNAM, México, $40 \mathrm{p}$.

Rueda, H., Macías, J.L., Arce, J.L., Gardner, J.E., Layer, P., 2013, The 31 ka rhyolitic Plinian to sub-Plinian eruption of Tlaloc Volcano, Sierra Nevada, central Mexico: Journal of Volcanology and Geothermal Research, 252, 73-91.

Sheth, H.C., Torres-Alvarado, I.S., Verma, S.P., 2002, What is the "Calcalkaline rock series"?: International Geology Review, 44, 686-701.

Siebe, C., Rodríguez-Lara, V., Schaaf, P., Abrams, M., 2004, Geochemistry, $\mathrm{Sr}-\mathrm{Nd}$ isotope composition, and tectonic setting of Holocene Pelado, Guespalapa, and Chichinautzin scoria cones, south of Mexico City: Journal of Volcanology and Geothermal Research, 130, 197-226.

Siebe, C., Arana-Salinas, L., Abrams, M., 2005, Geology and radiocarbon ages of Tláloc, Tlacotenco, Cuauhtzin, Hijo del Cuauhtzin, Teuhtli, and Ocusacayo monogenetic volcanoes in the central part of the Sierra Chichinautzin, México: Journal of Volcanology and Geothermal Research, 141, 225-243.

Siebe, C., Macías, J.L., 2004, Volcanic hazards in the Mexico City metropolitan area from eruptions at Popocatépetl, Nevado de Toluca, and Jocotitlán stratovolcanoes and monogenetic scoria cones in the Sierra Chichinautzin Volcanic Field: Field Guide, Penrose Conference, Neogene-Quaternary Continental Margin Volcanism, January 2004, State of Puebla, México: Boulder, Colorado, Geological Society of America, 77p.

Siebe, C., 2000, Age and archaelogical implications of Xitle volcano, southwestern Basin of Mexico City: Journal of Volcanology and Geothermal Research, 104, 45-64.

Solari, L.A., Gómez-Tuena, A., Bernal, J.P., Pérez-Arvizu, O., Tanner, M., 2010, U-Pb Zircon Geochronology with an Integrated LAICP-MS Microanalytical Workstation: Achievements in Precision and Accuracy: Geostandards and Geoanalytical Research, 34, 5-18.

Straub, S.M., Gómez-Tuena, A., Stuart, F.M., Zellmer, G.F., EspinasaPerena, R., Cai, M.Y., Iizuca, Y., 2011, Formation of hybrid arc andesites bebeath thick continental crust: Earth and Planetary Sciences Letters, 303, 337-347.

Straub, S.M., Gómez-Tuena, A., Zellmer, G.F., Espinasa-Perena, R., Stuart, F.M., Cai, M.Y., Langmuir, C.H., Martin Del Pozzo, A.L., Mesko, G.T., 2013, The processes of melt differentiation in arc volcanic rocks: Insights from OIB-type arc magmas in Central Mexican Volcanic Belt: Journal of Petrology, 54, 665-701.

Sun, S., McDonough, W., 1989, Chemical and isotopic systematics of oceanic basalts: implications for mantle compositions and processes, en Saunders, A., Norry, M., (Eds.), Magmatism in ocean basins: Geological Society of London, Special Publication, 42, 313-345.

Vazquez-Sanchez, E., Jaimes-Palomera, R., 1989, Geología de la Cuenca de México: Geofisica Internacional, 28, 133-174.

Verma, S.P., 2000, Geochemistry of the subducted Cocos plate and the origin of subduction-unrelated mafic volcanism at the volcanic front of the central Mexican Volcanic Belt, en Delgado, H., Aguirre-Díaz, G., Stock, J.M., (Eds), Cenozoic Tectonics and Volcanism of Mexico: Geological Society of America, Special Publication, 334, 195-222.

Waitz, P., 1910, Excursión geológica a la sierra de Santa Catarina: Boletín de la Sociedad Geológica Mexicana, 7, 1-7. 
Waitz, P., 1911, Excursión geológica a la parte poniente de la sierra de Santa Catarina: Boletín de la Sociedad Geológica Mexicana, 8, 1-8.

Wallace, P.J., Carmichael, I.S.E., 1999, Quaternary volcanism near the Valley of Mexico: Implications for subduction zone magmatism and the effects of crustal thickness variations on primitive magma compositions: Contribution to Mineralogy and Petrology, 135, 291-314.
Manuscrito recibido: Mayo 8, 2014

Manuscrito corregido recibido: Septiembre 25, 2014.

Manuscrito aceptado: Septiembre 30, 2014. 